\title{
The coalescence rate of double neutron star systems
}

\section{Citation}

Kalogera, V., R. Narayan, D. N. Spergel, and J. H. Taylor. 2001. “The Coalescence Rate of Double Neutron Star Systems." The Astrophysical Journal 556 (1): 340-56. https:// doi.org/10.1086/321583.

\section{Permanent link}

http://nrs.harvard.edu/urn-3:HUL.InstRepos:41384939

\section{Terms of Use}

This article was downloaded from Harvard University's DASH repository, and is made available under the terms and conditions applicable to Other Posted Material, as set forth at http:// nrs.harvard.edu/urn-3:HUL.InstRepos:dash.current.terms-of-use\#LAA

\section{Share Your Story}

The Harvard community has made this article openly available.

Please share how this access benefits you. Submit a story.

Accessibility 


\title{
The Coalescence Rate of Double Neutron Star Systems
}

\author{
V. Kalogera ${ }^{1}$, R. Narayan ${ }^{1}$, D.N. Spergel ${ }^{2,3}$, and J.H. Taylor ${ }^{4}$
}

\begin{abstract}
We estimate the coalescence rate of close binaries with two neutron stars (NS) and discuss the prospects for the detection of NS-NS inspiral events by ground-based gravitational-wave observatories, such as LIGO. We derive the Galactic coalescence rate using the observed sample of close NS-NS binaries (PSR B1913+16 and PSR B1534+12) and examine in detail each of the sources of uncertainty associated with the estimate. Specifically, we investigate (i) the dynamical evolution of NS-NS binaries in the Galactic potential and the vertical scale height of the population, (ii) the pulsar lifetimes, (iii) the effects of the faint end of the radio pulsar luminosity function and their dependence on the small number of observed objects, (iv) the beaming fraction, and (v) the extrapolation of the Galactic rate to extragalactic distances expected to be reachable by LIGO. We find that the dominant source of uncertainty is the correction factor (up to $\simeq 200$ ) for faint (undetectable) pulsars. All other sources are much less important, each with uncertainty factors smaller than 2. Despite the relatively large uncertainty, the derived coalescence rate is consistent with previously derived upper limits, and is more accurate than rates obtained from population studies. We obtain a most conservative conclusion that the detection rate for LIGO II lies in from range from 2 events per year up to at least 300 events per year or even possibly in excess of 1000 events per year.
\end{abstract}

\section{INTRODUCTION}

The importance of close binaries with two neutron stars (NS-NS) for gravitational wave physics was established a few years after the discovery of the prototype NS-NS system, the binary PSR B1913+16 (Hulse \& Taylor 1975), with the measurement of orbital decay at a rate consistent with gravitational wave emission as predicted by general relativity (at a $3 \times 10^{-3}$ accuracy level) (Taylor \& Weisberg 1982; 1989; 1999, unpublished). This orbital decay is expected

\footnotetext{
${ }^{1}$ Harvard-Smithsonian Center for Astrophysics, 60 Garden St., Cambridge, MA 02138; vkalogera and rnarayan@cfa.harvard.edu

${ }^{2}$ Princeton University Observatory, Princeton, NJ 08544; dns@astro.princeton.edu

${ }^{3}$ WM Keck Distinguished Visiting Professor of Astrophysics, IAS, Princeton NJ 08540

${ }^{4}$ Joseph Henry Laboratories and Physics Department, Princeton University, Princeton, NJ 085544; joe@pulsar.princeton.edu
} 
to end catastrophically with the merger of the two neutron stars as the binary orbital separation becomes comparable to the NS radii (for a recent review, see Rasio \& Shapiro 1999). Such inspiral events and the final mergers could possibly be detected as gravitational wave sources by the currently built ground-based laser interferometers, such as LIGO and VIRGO. With the upcoming completion of these observatories increased interest has focused on close binaries with two compact objects.

Assessment of the detectability of binary compact inspiral events depends both on the strength of the gravitational wave signal and the frequency of such mergers out to extragalactic distances. Based on the expected sensitivity of LIGO I and II, a NS-NS inspiral could be detected out to $20 \mathrm{Mpc}$ and $350 \mathrm{Mpc}$, respectively (Gustafson et al. 1999). Predictions for the expected detection rates can be made based on estimates of the Galactic coalescence rate and its extrapolation to these maximum distances of interest. Such Galactic estimates have been obtained in two different ways so far: empirically, based on the observed NS-NS sample, and purely theoretically, based on our understanding of NS-NS formation.

Binary neutron stars can be discovered in radio pulsar surveys, if one of the two neutron stars emits radio pulses. In the last two decades, four NS-NS binaries in addition to PSR B1913+16 have been discovered by sensitive pulsar searches: PSR B1534+12 (Wolszczan 1991), PSR B2127+11C (Prince et al. 1991), PSR J1518+4904 (Nice et al. 1996), and PSR J1811-1736 (Lyne et al. 2000). Of these four, only the first two are coalescing binaries, along with PSR B1913+16, i.e., have tight enough orbits so that the two neutron stars will coalesce within $10^{10} \mathrm{yr}$. One other binary pulsar, PSR B2303+46 (Lyne \& Bailes 1990), was classified as a NS-NS binary for many years, until it was optically identified (van Kerkwijk \& Kulkarni 1999) and is now thought to be a binary pulsar with a massive white dwarf companion. Recently, another binary pulsar, PSR J1141-6545, with a relatively short coalescence time was discovered by the ongoing Parkes multibeam pulsar survey (Lyne et al. 2000) and was also initially considered to be a NS-NS candidate. However, the low measured total mass of the system points to a white dwarf companion to the pulsar rather than a neutron star (Kaspi et al. 2000). In the analysis presented in this paper, we include the two established NS-NS binaries with short merger times found in the Galactic field, i.e., PSR B1913+16 and PSR B1534+12. We do not include PSR B2127+11C, which is found in a globular cluster, for two main reasons: (i) its association with globular clusters implies very different formation history (dominated by dynamical interactions) and detection selection effects, and therefore our analysis cannot be applied to such systems, and (ii) based on the globular cluster space density, the contributions of cluster systems to the coalescence rate has been estimated by Phinney (1991) to be very small (by more than a factor of 10).

These discoveries have contributed to our knowledge of the properties of NS-NS binaries and allow empirical estimates of the coalescence rate, based on a quantitative analysis of the selection effects relevant to pulsar surveys (e.g., Narayan et al. 1991; Phinney 1991). Also, over the years, a theoretical understanding of the NS-NS formation process has developed and estimates of their birth rate have been obtained based on theoretical calculations of binary evolution (e.g., Lipunov 
et al. 1997; Fryer et al. 1998; Portegies-Zwart \& Yungel'son 1998; Bethe \& Brown 1998; Fryer et al. 1999; Bulik et al. 1999; Grishchuk et al. 2001). Still, however, serious uncertainties remain that hamper settling the question of whether close NS-NS systems are formed at adequately high rates to provide a reasonable LIGO detection rate (at least a few events per year). At present, theoretical estimates cover a wide range of values (three to four orders of magnitude; see Kalogera 2000a) and appear to have a rather limited predictive power 5 .

Neutron star coalescence has also been discussed as a possible central engine of gamma-ray bursts (e.g., Paczynski 1986; Eichler et al. 1989; Narayan et al. 1992) and a production site of r-process elements (Eichler et al. 1989; Rosswog et al. 1999). Among other issues, accurate estimates of merger frequencies could be useful in examining these proposed associations and possibly constraining the degree of beaming in gamma-ray burst emission (e.g., Belczynski et al. 1999).

In this paper we focus on estimates of the Galactic birth rate of coalescing NS-NS binaries based on the current observed sample. In $\S 2$, we give a brief overview of such empirical estimates and the main steps involved in the calculations. In $\S 3$, we describe our derivation of the NS-NS coalescence rate in our Galaxy, addressing each of the uncertainties involved: NS-NS scale height in the Galaxy, system lifetimes, corrections for the faint end of the radio pulsar luminosity function, beaming, and pulse smearing due to orbital acceleration. In $\S 4$, we extrapolate our Galactic estimate to extragalactic distances relevant to LIGO I and II. In $\S 6$, we present our conclusions on the NS-NS coalescence rate, the associated uncertainty, and the expected LIGO detection rates. We also compare our results to the theoretical estimates derived based on binary evolution calculations.

\section{PREVIOUS ESTIMATES OF THE GALACTIC NS-NS COALESCENCE RATE}

Estimates of the NS-NS coalescence rate in our Galaxy can be obtained using the observed properties of the radio pulsars in such binaries and the characteristics of radio pulsar surveys. Based on these two elements, a model can be constructed accounting for pulsar selection effects and the detectability of the observed pulsars throughout the Galaxy. For each pulsar observed in a coalescing NS-NS system, a scale factor, $\mathcal{S}$, can be calculated (e.g., Narayan 1987). It is defined as the inverse of the fraction of the Galactic volume (weighted by the radio-pulsar spatial distribution in the Galaxy), within which pulsars with properties identical to those of the observed

\footnotetext{
${ }^{5}$ However, taking into account multiple observational constraints on the absolute calibration of population synthesis models can significantly improve these estimates. See, for example, Belczynski, Kalogera, \& Bulik 2000.
} 
pulsar could be detected by any of the pulsar surveys completed so far,

$$
\mathcal{S}=\frac{\int_{V_{G}} F_{p}(R, Z) d V}{\int_{V_{D}} F_{p}(R, Z) d V},
$$

where $F_{p}$ describes the radial $(R)$ and vertical $(Z)$ distribution of pulsars in the Galaxy $\left(F_{p}\right.$ is usually assumed to be axisymmetric and separable in $R$ and $Z$ ), $V_{G}$ is the Galactic volume, and $V_{D}$ is the volume within which each observed pulsar could be detected by the pulsar surveys. The scale factor $\mathcal{S}$ is a measure of how many more pulsars like those already detected in coalescing NS-NS binaries exist in the Galaxy. The coalescence rate is then estimated using these scale factors divided by estimates for the lifetime of each radio pulsar summed up over all detected coalescing NS-NS. This estimate can be further corrected for a fraction of undetected pulsars either because the pulsar beam does not intersect our line of sight (beaming fraction), or because they are too faint to be detected even by the most sensitive surveys conducted so far, or because the binary orbital periods are so short that the pulses are smeared due to orbital acceleration.

This method was first applied by Narayan et al. (1991) and Phinney (1991) to obtain empirical estimates of the NS-NS coalescence rate in the Galaxy. Narayan et al. (1991) adopted a Gaussian form for $F_{p}\left(\propto \exp \left[-(R / 8 \mathrm{kpc})^{2}-\left(Z / Z_{0}\right)^{2} / 2\right]\right.$, where $Z_{0}$ is the vertical scale height), and Phinney (1991) adopted a constant pulsar density in a cylinder of radius $R_{0}=12 \mathrm{pc}$ and half-height $Z_{0}$. Both groups considered the same pulsar surveys and made similar assumptions about the lifetimes of PSR B1913+16 and PSR B1534+12. They assumed that the lifetime of a pulsar binary is equal to the sum of the pulsar characteristic age $\left(\tau_{c}=P / 2 \dot{P}\right)$ and the binary merger time $T_{m}$, i.e., the time it will take for the binary to coalesce through gravitational radiation emission. Without any further corrections for beaming, the faint end of the pulsar luminosity function, or pulse smearing due to orbital acceleration, both studies obtained an estimate of the Galactic NS-NS coalescence rate equal to $10^{-6} \mathrm{yr}^{-1}$ assuming $Z_{0}=1 \mathrm{kpc}$.

These early estimates have been subsequently revised as more of the Galactic volume was searched for pulsars. Curran \& Lorimer (1995) included more pulsar surveys, which did not lead to new NS-NS discoveries. However, they did not include the Green Bank Northern Sky Survey that discovered PSR J1518+4904. They assumed the same lifetimes as Narayan et al. (1991) and Phinney (1991), and investigated in more detail the dependence of the derived rate on the assumed radial and vertical pulsar distribution in the Galaxy. They found that the scale factors decrease by a factor of two if a radial distribution with a strong deficit of pulsars in the inner Galactic region (Johnston 1994) is assumed. The dependence on the scale height was found to be linear assuming an exponential vertical distribution $\left(\propto \exp \left(-|Z| / Z_{0}\right)\right.$. For $Z_{0}=0.5 \mathrm{kpc}$, a radial distribution similar to that assumed by Narayan et al. (1991), and without any further corrections, they obtain a rate estimate of $\simeq 2 \times 10^{-7} \mathrm{yr}^{-1}$. The primary reasons for this significant reduction compared to the earlier estimates were (i) the use of a different pulsar distance model, which leads to a higher luminosity for PSR B1534+12, and (ii) the lack of any new discoveries of NS-NS binaries with the additional pulsar surveys. Curran \& Lorimer (1995) also included a beaming correction of a factor of 3 and a 10-fold correction for the undetected pulsars at the faint end of the luminosity 
function (extrapolation down to $1 \mathrm{mJy} \mathrm{kpc}^{2}$ ) raising the estimate to $\simeq 6 \times 10^{-6} \mathrm{yr}^{-1}$.

Van den Heuvel \& Lorimer (1996) reconsidered the NS-NS lifetimes and argued that the binary merger time should be replaced by the time in which the luminosity of the observed pulsars drops below a detection threshold (when the magnetic dipole energy loss rate reaches that of a typical normal pulsar after $10 \mathrm{Myr}$ ). This strongly model-dependent modification was applied along with the 10-fold correction for the faint pulsars introduced by Curran \& Lorimer (1995). Based on this van den Heuvel \& Lorimer (1996) concluded that the NS-NS coalescence rate is raised by a factor of 2.7 compared to the Curran \& Lorimer (1995) result. More recently, measurements of relativistic orbital parameters (Stairs et al. 1998) have provided us with a precise distance measurement for PSR B1534+12, which led to a downward revision of the van den Heuvel \& Lorimer (1996) rate to $\simeq 6.5-8.5 \times 10^{-6} \mathrm{yr}^{-1}$.

Arzoumanian et al. (1998) examined once again the issue of the NS-NS lifetimes. They argued that a better estimate of the present age can be obtained based on the pulsar spin-down history, assuming that their life started at the spin-up line in the period-period derivative plane for pulsars (uncertainties on the definition of the spin-up line lead to age variations smaller than a factor of 2). Concerning the remaining lifetime of the binaries, they adopted the "luminosity-evolution" lifetime suggested by van den Heuvel \& Lorimer (1996), but also considered pulsar selection effects against short-period binaries, because of pulse smearing (it becomes important only for PSR B1913+16). Using the scale factors derived by Curran \& Lorimer (1995), they revised the rate to $2.7 \times 10^{-7} \mathrm{yr}^{-1}$, and to $8 \times 10^{-6} \mathrm{yr}^{-1}$ when they included the same corrections for beaming and faint pulsars. In addition, Arzoumanian et al. (1998) obtained another rate estimate based on (i) a revised (increased by more than a factor of 3) total Galactic volume $V_{G}$, (ii) a revised detection volume $V_{D}$ integrating out to maximum detection distances and over the pulsar luminosity function (in this way, a correction for the faint end of the luminosity function was incorporated to some extent but no weighting based on the Galactic distribution of pulsars was applied), and (iii) an average lifetime for all NS-NS systems, since no individual scale factors were calculated. They obtained a much lower estimate of $\simeq 2 \times 10^{-7} \mathrm{yr}^{-1}$ (this does not include any beaming correction).

Evans et al. (1999) followed a rather different approach and argued that the calculations of the detection volume and the pulsar lifetimes cannot be performed separately because pulsar luminosity evolution affects both simultaneously. Instead, for each of the two coalescing NS-NS systems, they calculated an average visibility factor. This factor accounts for the motion of such systems in the Galaxy, given a range of initial velocities and taking into account the selection effects associated with all pulsar surveys to date. The sum of the reciprocals of these visibility factors is an estimate of the coalescence rate. Preliminary results yield an estimate of $7 \times 10^{-8} \mathrm{yr}^{-1}$ (without any beaming correction). 


\section{GALACTIC NS-NS COALESCENCE RATE AND UNCERTAINTIES}

In what follows we present our derivation of the Galactic NS-NS coalescence rate addressing each one of the elements that enter the calculation. For some of these elements, we use the results of earlier studies but we examine in more detail the associated uncertainties and the sensitivity of our estimate to various factors.

\subsection{Scale Factors}

The calculation of scale factors depends on the pulsar surveys completed so far and the assumed spatial distribution of NS-NS binaries. Curran \& Lorimer (1995) have considered all the pulsar surveys to date except for the ongoing Parkes Multibeam survey (Lyne et al. 2000). They also studied the dependence of the scale factors on the assumed radial distribution. Combined with the results obtained by Narayan et al. (1991) and Phinney (1991), it becomes evident that the scale factors are not highly sensitive to such variations, unless the radial distribution is strongly modified (large deficit of pulsars in the center of the Galaxy, see Johnston 1994). Here, we use the scale-factor results obtained by Curran \& Lorimer (1995), for a Gaussian radial distribution with a scale length $R_{0}=4.8 \mathrm{kpc}$ (higher by a factor of $\simeq 2$ - within their statistical errors relative to the results obtained when the Johnston 1994 radial distribution is assumed; see Table 1 and Figure 2 in Curran \& Lorimer 1995) and an assumed scale height $Z_{0}=0.5 \mathrm{kpc}$. We also take into account the significant reduction of the scale factor for PSR B1534+12, caused by the relativistic measurement of its distance (Stairs et al. 1998). Depending on the scale height of the NS-NS population this reduction factor could be as low as 2.5 (if $Z_{0}<1 \mathrm{kpc}$ ) and as high as 4 (if $Z_{0}>1 \mathrm{kpc}$ ). Since Curran \& Lorimer (1995) derived the scale factors assuming $Z_{0}=0.5 \mathrm{kpc}$, we apply a correction factor of 2.5 . So, the scale factors we use in our rate derivation are $\mathcal{S}=40$ for PSR B1913+16 and $\mathcal{S}=130$ for PSR B1534+12. Statistical errors associated with these values are $\simeq 5-6 \%$. These scale factors will be further modified by our calculation of $Z_{0}$ based on the linear dependence derived by Curran \& Lorimer (1995).

\subsection{Vertical Scale Height}

Narayan et al. (1991) found that the coalescence rate is roughly linearly proportional to the scale height, $Z_{0}$, of the Galactic NS-NS population. This dependence originates mainly from the $Z_{0}$ dependence of the total Galactic volume weighted by the NS-NS spatial distribution (see equation [1]) . However, due to the spatial distribution weighting, the scale height enters the calculation of the detection volume as well. Curran \& Lorimer (1995) examined this dependence in more detail and found that the scale factor (summed up for the three binaries PSR B1913+16, PSR B1534+12,

and PSR B2303+46) has the following linear dependence: $\mathcal{S}_{\text {total }} \propto 0.1\left(Z_{0} / \mathrm{kpc}\right)+0.19$, assuming an exponential vertical distribution: $\propto \exp \left(-|Z| / Z_{0}\right)$. The value of $Z_{0}$ is usually assumed to lie 
in the range $0.5-1 \mathrm{kpc}$. However, we undertake a more detailed study and we calculate it based on realistic models for the motion of coalescing NS-NS binaries in the Galaxy. In what follows we describe our approach in detail.

\subsubsection{Physical Model and Numerical Method}

The formation history of coalescing NS-NS binaries involves a number of evolutionary stages including two supernova explosions. The associated mass loss and birth kicks imparted to the neutron stars affect the center-of-mass velocities $\left(V_{\mathrm{CM}}\right)$ of the binaries. The post-supernova velocities are closely related to the relative orbital velocity $\left(V_{r}\right)$ in the pre-supernova orbit and a strict upper limit of $2 V_{r}$ to $V_{\mathrm{CM}}$ can be derived (e.g., Brandt \& Podsiadlowski 1995; Kalogera 1996). The binary orbits of NS-NS progenitors before the first supernova (SN) explosion are so wide that the typical post-explosion center-of-mass velocities are $V_{\mathrm{CM}} \lesssim 50-75 \mathrm{~km} \mathrm{~s}^{-1}$, i.e., significantly lower than the typical velocities associated with Galactic rotation. However, just prior to the second SN explosion, progenitors of coalescing NS-NS are expected to have orbits tight enough that typical post-explosion velocities reach or even exceed $500 \mathrm{~km} \mathrm{~s}^{-1}$. Such high velocities alter significantly the kinematic evolution of the NS-NS population and lead to vertical scale heights much larger than that of their initial progenitors (the typical scale height of massive stars is $\sim 50-75 \mathrm{pc}$ ).

In what follows we consider NS-NS progenitors just before the second SN explosion. According to all NS-NS formation mechanisms discussed in the literature (see Fryer \& Kalogera 1997 for a brief overview), the binaries at this stage consist of the first NS and a helium-rich companion (the core of the original secondary, i.e., less massive star, in the binary) in circular orbits. In fact, (i) circular pre-SN] orbits with sizes $\lesssim 50-100 \mathrm{R}_{\odot}$, and (ii) helium-star companions with masses in the range $3-10 \mathrm{M}_{\odot}$ are necessary for the formation of tight, coalescing NS-NS binaries (see Fryer \& Kalogera 1997; Fryer et al. 1999; Wex et al. 2000; Fryer 1999, private communication). Such progenitor properties are achieved through common-envelope evolution, which has to occur some time between the two supernova explosions. We assume that isotropic birth kicks of a certain magnitude distribution are imparted to the newborn neutron stars and we calculate the $V_{\mathrm{CM}}$ distribution for the subset of post-SN systems that (i) remain bound after the explosion, and (ii) have binary properties (post-SN orbital separations and eccentricities) such that their merger times do not exceed $10^{10} \mathrm{yr}$.

For given values of the helium-star mass $M_{0}$, the pre-SN orbital separation $A_{0}$, and the isotropic kick magnitude $V_{k}$, we can use conservation laws of energy and angular momentum for the system and derive expressions for the post-SN orbital semi-major axis $A$ and eccentricity $e$.

\footnotetext{
${ }^{6}$ From here and on, SN refers specifically to the second supernova explosion in the formation history of NS-NS binaries
} 
The center-of-mass velocity $V_{\mathrm{CM}}$ after the explosion can also be calculated (see Hills 1983; Brandt \& Podsiadlowski 1995; Kalogera \& Lorimer 2000):

$$
\begin{gathered}
A=\frac{\beta A_{0}}{2 \beta-u_{k}^{2} \sin ^{2} \theta-\left(u_{k} \cos \theta+1\right)^{2}} \\
1-e^{2}=\frac{1}{\beta^{2}}\left[u_{k}^{2} \sin ^{2} \theta \cos ^{2} \phi+\left(u_{k} \cos \theta+1\right)^{2}\right]\left[2 \beta-u_{k}^{2} \sin ^{2} \theta-\left(u_{k} \cos \theta+1\right)^{2}\right] \\
\left(\frac{V_{\mathrm{CM}}}{V_{\mathrm{orb}}}\right)^{2}=\frac{M_{\mathrm{NS}}\left(M_{0}-M_{\mathrm{NS}}\right)}{2\left(M_{0}+M_{\mathrm{NS}}\right)^{2}} \frac{A_{0}}{A}+\frac{M_{0}}{2\left(M_{0}+M_{\mathrm{NS}}\right)}\left(\frac{V_{k}}{V_{\mathrm{orb}}}\right)^{2}+\frac{\left(M_{0}-M_{\mathrm{NS}}\right)\left(M_{0}-2 M_{\mathrm{NS}}\right)}{2\left(M_{0}+M_{\mathrm{NS}}\right)^{2}} .
\end{gathered}
$$

In the above equation $V_{\text {orb }} \equiv\left[G\left(M_{\mathrm{NS}}+M_{0}\right) / A_{0}\right]^{1 / 2}$ is the pre-SN relative orbital velocity, $\beta \equiv\left(M_{\mathrm{NS}}+M_{\mathrm{NS}}\right) /\left(M_{\mathrm{NS}}+M_{0}\right)$, and angles $\theta$ and $\phi$ describe the direction of the kick: $\theta$ is the polar angle from the pre-SN orbital velocity vector of the exploding He-star and ranges from $0-\pi$ (at $\theta=0, \vec{V}_{k}$ and $\vec{V}_{\text {orb }}$ are aligned); $\phi$ is the azimuthal angle in the plane perpendicular to $\vec{V}_{\text {orb }}$ (i.e., $\theta=\pi / 2$ ) and ranges from $0-2 \pi$ (at $\theta=\pi / 2$ and $\phi=0$ or $\phi=\pi$, the kick component points along or opposite to the angular momentum axis of the pre-SN orbital plane, respectively; see Figure 1 in Kalogera 2000b). For a given pair of $A$ and $e$, the merger time scale $T_{\mathrm{m}}$ is given by (Peters \& Mathews 1963; Shapiro \& Teukolsky 1983) as

$$
T_{\mathrm{m}} \equiv\left(\frac{d \ln E_{\mathrm{orb}}}{d t}\right)^{-1} \simeq 300 \operatorname{Myr}\left(\frac{M_{\mathrm{NS}}}{M_{\odot}}\right)^{-3}\left(\frac{A}{R_{\odot}}\right)^{4}\left(1-e^{2}\right)^{7 / 2}\left(1+\frac{73}{24} e^{2}+\frac{37}{96} e^{4}\right)^{-1} .
$$

We note that this is just the time scale associated with the orbital energy loss rate due to gravitational radiation, and not the time interval over which a binary of given $A$ and $e$ will merge (this can be calculated by solving the coupled equations for the rate of change of both orbital separation and eccentricity; Peters \& Mathews 1963). Comparison of these two quantities shows that, because orbital shrinkage rapidly accelerates with time, the merger time scale typically overestimates the actual merger time by factors of $\lesssim 4$ (for circular orbits, the difference is exactly a factor of 4; in the cases of PSR B1913+16 and PSR B1534+12, the factors are 1.87 and 3.3, respectively). In what follows we use the merger time scale, $T_{\mathrm{m}}$. However, we examine the effect of this difference on the derived scale heights. We find that if we decrease the calculated $T_{\mathrm{m}}$ by a uniform factor of 3 , the scale height decreases (as expected qualitatively) by less than $2.5 \%$.

We first derive the distribution function of $A$ and $e$ from the distribution of the direction angles, $\theta$ and $\phi$, for an isotropic kick with a simple Jacobian transformation

$$
\begin{aligned}
F(A, e)= & F(\theta, \phi) J\left(\frac{\theta, \phi}{A, e}\right)=\frac{\sin \theta}{2} \frac{1}{2 \pi} J\left(\frac{\theta, \phi}{A, e}\right) \\
= & \frac{\beta^{2} e V_{\text {orb }}}{2 \pi V_{k} A}\left[\beta\left(1-e^{2}\right) A / A_{0}-\left(\frac{2 \beta-\beta A_{0} / A-\left(V_{k} / V_{\text {orb }}\right)^{2}-1}{2}+1\right)^{2}\right]^{-1 / 2} \\
& \times\left[2 \beta-\beta A_{0} / A-\beta\left(1-e^{2}\right) A / A_{0}\right]^{-1 / 2} .
\end{aligned}
$$


With a similar transformation between $A$ and $V_{\mathrm{CM}}$ and integration over $e$ we can obtain numerically

$$
F\left(V_{\mathrm{CM}}\right)=\int_{0}^{1} F(A, e) J\left(\frac{A, e}{V_{\mathrm{CM}}, e}\right) d e .
$$

However, the dynamical evolution of the whole population in the Galactic potential depends not only on the initial velocity distribution but also the pulsar lifetimes. Any strong correlation between the two quantities can affect the final vertical distribution of the population. In fact, from studies of the effects of SN kicks on binary properties (e.g., Kalogera 1996), we know that post-SN binaries with tight or significantly eccentric orbits tend to acquire large center-of-mass velocities. Such tight and eccentric orbits, however, will typically have short merger time scales. Consequently, systems with large initial velocities, which could drive the population to high vertical scale heights, typically have short timescales, which counteracts their expansion in the Galaxy. To include all these effects rigorously in our calculation, we derive the two-dimensional distribution function of $V_{\mathrm{CM}}$ and $T_{\mathrm{m}}$, for given $V_{k}, M_{0}, A_{0}$ :

$$
\begin{aligned}
F\left(V_{\mathrm{CM}}, T_{\mathrm{m}} ; V_{k}, M_{0}, A_{0}\right)= & F(A, e) J\left(\frac{A, e}{V_{\mathrm{CM}}, T_{\mathrm{m}}}\right) \\
= & \frac{4 A^{2} V_{\mathrm{CM}}}{A_{0} V_{\mathrm{orb}}^{2} T_{\mathrm{m}}} \frac{\left(M_{0}+M_{\mathrm{NS}}\right)^{2}}{M_{\mathrm{NS}}\left(M_{0}-M_{\mathrm{NS}}\right)} F\left[A\left(V_{\mathrm{CM}}\right), e\left(V_{\mathrm{CM}}, T_{\mathrm{m}}\right)\right] \\
& \times\left[\left(2 \frac{73}{24} e+4 \frac{37}{96} e^{3}\right)\left(1+\frac{73}{24} e^{2}+\frac{37}{96} e^{4}\right)^{-1}+\frac{7 e}{1-e^{2}}\right]^{-1} .
\end{aligned}
$$

All the derivatives involved in the Jacobian transformations appearing in equations (6)-(8) are calculated analytically using equations (2)-(5). From equation (4), we obtain $A$ as a function of $V_{\mathrm{CM}}$ analytically, and from equation (5), we obtain that of $e$ as a function of $T_{\mathrm{m}}$ numerically. The next step is to convolve the above distributions with a kick magnitude distribution as well as the distribution functions of $M_{0}$ and $A_{0}$, which we assume to be separable:

$$
\begin{aligned}
F\left(V_{\mathrm{CM}}, T_{\mathrm{m}}\right)=\int_{M_{0}} \int_{V_{k}} \int_{A_{0}} F\left(V_{\mathrm{CM}}, T_{\mathrm{m}} ; V_{k}, M_{0}, A_{0}\right) \times \\
f_{A_{0}}\left(A_{0}\right) f_{M_{0}}\left(M_{0}\right) f_{V_{k}}\left(V_{k}\right) d A_{0} d V_{k} d M_{0} .
\end{aligned}
$$

The ranges of integration over $A_{0}$ are dependent on $V_{k}$ and $M_{0}$ and are determined by the requirement that the post-SN binaries are coalescing. The range of integration over $M_{0}$ is restricted to those helium star progenitors that are expected to end their lives as neutron stars (instead of black holes), i.e., $2-10 \mathrm{M}_{\odot}$ (Fryer 1999 and private communication). It turns out that ranges of interest for $A_{0}$ and $M_{0}$ are narrow enough that our final results are not at all sensitive to the details of the chosen distribution functions. In what follows, we adopt an $A_{0}$-distribution flat in $\log A_{0}$ and a power-law function for $f_{M_{0}}\left(M_{0}\right) d M_{0} \propto M_{0}^{-\alpha} d M_{0}$ with $\alpha$ in the range $1-3$ (these choices are motivated by typical assumptions about the characteristics of the primordial binary population, e.g., Kalogera \& Webbink 1998; Portegies-Zwart \& Yungel'son 1998). The two-dimensional distribution can be also integrated over $V_{\mathrm{CM}}$ or $T_{\mathrm{m}}$ to obtain one-dimensional distributions (as described in the next section). 
We consider a population of NS-NS progenitors distributed in the Galaxy with a Gaussian radial distribution in azimuthal symmetry. The population is assumed for simplicity to lie in the Galactic plane. This assumption is motivated by the very small (much smaller than the expected scale height of NS-NS binaries) scale height of the Galactic massive star population and the small (smaller than typical Galactic rotational velocities) center-of-mass velocities after the first supernova explosion. We have checked and confirmed the validity of this assumption using our numerical results and studying models where the initial population is assumed to lie at $Z= \pm 100 \mathrm{pc}$. The resulting distributions of binaries in the vertical direction are identical to an accuracy of $\sim 0.1 \%$ or better. For the orbit calculations, we use the Galactic gravitational potential of Kuijken \& Gilmore (1989). We make sure that the Galactic rotation curve used in the calculation is consistent with the chosen potential.

We follow the dynamical evolution of the NS-NS population in the five-dimensional phase space of two spatial dimensions (radial $R$ and vertical $Z$ positions) and three velocity components $\left(u_{\Phi}, u_{R}, u_{Z}\right)$, all defined in a reference frame of cylindrical coordinates centered on the Galactic center. The distribution function that describes the binary population at $t=0$ is actually six-dimensional because it includes the merger time scale $\left(T_{\mathrm{m}}\right)$, although it does not evolve with time:

$$
F_{0}\left(R, Z, u_{\Phi}, u_{R}, u_{Z}, T_{\mathrm{m}}\right)=f_{R}(R) \delta(Z) f_{u}\left(u_{\Phi}, u_{R}, u_{Z}, T_{\mathrm{m}}\right)
$$

i.e., we assume separation of variables for the spatial components and that the initial $Z$-distribution is a delta function in the Galactic plane $(Z=0)$. The initial velocity distribution $f_{u}$ is calculated using the distributions of post-SN center-of-mass velocities for coalescing NS-NS binaries $F\left(V_{\mathrm{CM}}, T_{\mathrm{m}}\right)$ and assuming that they are imparted to the binaries isotropically with respect to their Galactic rotation velocities (the latter depend on their radial position and the assumed Galactic potential).

We first set up a grid of initial phase-space positions and we calculate the Galactic orbits in the chosen fivel-dimensional phase space as a function of time, for a maximum time of $10^{10} \mathrm{yr}$. We have tested numerically the initial grid and adopted one of high enough density so that numerical artifacts in the analysis are avoided. In the present study, we are interested in the vertical distribution of the coalescing NS-NS binaries. For a given Galactic gravitational potential, the vertical distance $Z$ is a unique function of the time $t$, for which they have been moving in the Galaxy, and the initial phase space positions $Z^{\prime}=Z\left(t, R, u_{\Phi}, u_{R}, u_{Z}\right)$. Using exactly this functional dependence, which is calculated numerically by calculating the orbits in phase space, we can calculate the $Z$-distribution at a given time $t$ (for a grid of $t$ values up to $10^{10} \mathrm{yr}$ ). The calculation involves a one-dimensional Jacobian transformation and a multiple numerical

\footnotetext{
${ }^{7}$ Note that azimuthal symmetry is conserved since both the gravitational potential and the initial spatial distribution are axisymmetric
} 
integration over the initial phase space variables:

$$
F_{t}\left(Z^{\prime}, T_{\mathrm{m}}\right)=\int_{R} \int_{u_{\Phi}} \int_{u_{R}} f_{R}(R) f_{u}\left(u_{\Phi}, u_{R}, u_{Z}, T_{\mathrm{m}}\right)\left[\left(\frac{\partial Z^{\prime}(t)}{\partial u_{Z}}\right)_{R, u_{\Phi}, u_{R}}\right]^{-1} d u_{R} d u_{\Phi} d R .
$$

The integration limits are chosen based on the shape of the distribution functions $f_{R}$ and $f_{u}$ and the range of values they cover (for $f_{u}$, we take into account the $V_{\mathrm{CM}}$ distribution calculated for each model and the contribution of Galactic rotation velocities as a function of $R$ ). We have performed numerical tests to examine the effects of the chosen limits and we make sure that their effect on the integral lies well below $1 \%$.

We are interested in the $Z$-distribution of coalescing NS-NS binaries at present, calculated taking into account (i) the star formation history of the Galaxy and (ii) the distribution of merger time scales, which can be significantly shorter than $10^{10} \mathrm{yr}$. Assuming a constant (per unit time) star formation rate, $C_{\mathrm{SFR}}$, over the age of the Galaxy, the distribution function describing the NS-NS population, i.e., number of systems per unit $Z^{\prime}$, per unit merger time, and per unit formation time over the age of the Galaxy is given by: $F_{t}\left(Z^{\prime}, T_{\mathrm{m}}\right) \times C_{\mathrm{SFR}}$. To obtain the $Z$-distribution of coalescing NS-NS binaries at present, we have to integration the latter distribution function over all merger times, $T_{m}$, and all formation times, $T_{F}$. Since $t$ is the time since the second supernova explosion, their formation time is: $T_{F}=10^{10}-t$. The range of integration for $T_{F}$ is determined by the fact that $t$ cannot exceed the associated merger time scale $\left(t \leq T_{\mathrm{m}}\right)$. To obtain the $Z$-distribution at present, we then have to integrate over all $T_{\mathrm{m}}$, yielding:

$$
\begin{aligned}
F\left(Z^{\prime}\right) & =C_{\mathrm{SFR}} \int_{T_{\mathrm{m}}=0}^{T_{\mathrm{m}}=10^{10}} \int_{T_{F}=10^{10}-T_{\mathrm{m}}}^{T_{F}=10^{10}} F_{t}\left(Z^{\prime}, T_{\mathrm{m}}\right) d T_{F} d T_{\mathrm{m}} \\
& \propto \int_{T_{\mathrm{m}}=0}^{T_{\mathrm{m}}=10^{10}} \int_{t=0}^{t=T_{\mathrm{m}}} F_{t}\left(Z^{\prime}, T_{\mathrm{m}}\right) d t d T_{\mathrm{m}} .
\end{aligned}
$$

Since the goal of this calculation is to obtain the scale height $Z_{0}$, we do not have to worry about the absolute normalization of the distribution function (e.g., the value of $C_{\mathrm{SFR}}$ ).

We wish to note that the computational method of distribution-function evolution in an appropriate phase space is very different from Monte Carlo methods that are more widely used for these types of problems (binary population synthesis and dynamical evolution of populations in a fixed potential). Our choice of methods has been driven by their high numerical accuracy at a

relatively low computational cost. A general discussion and comparison can be found in Kalogera \& Belczynski (2000). Specific to the problem of dynamical evolution, an additional advantage of the method is that it allows us to calculate the needed grid of orbits only once (this is the most computationally demanding part of the calculation) and then use it in the parameter study.

\subsubsection{Results}

We calculate the distribution of coalescing NS-NS binaries in birth center-of-mass velocities, merger time scales, and vertical distance from the Galactic plane, for different kick-magnitude 
distributions: a set of Maxwellian distributions with $\sigma=100,200,300,400 \mathrm{~km} \mathrm{~s}^{-1}$ and a "Paczynski-like" (Paczynski 1990) distribution with a large fraction of small-magnitude kicks: $f_{k}\left(V_{k}\right) \propto\left(1+u^{2}\right)^{-1}$, where $u=V_{k} / 600 \mathrm{~km} \mathrm{~s}^{-1}$. These distributions are consistent with studies of the radio-pulsar population (e.g., Cordes \& Chernoff 1997; Hartman 1997; Fryer et al. 1998), but also cover an adequately large range of average kick magnitudes.

A set of distribution functions (normalized to unity) of $V_{\mathrm{CM}}$ are shown in Figure 1, for a specific helium-star mass $\left(M_{0}=4 \mathrm{M}_{\odot}\right.$, as an example). It is evident that birth $V_{\mathrm{CM}}$ tends to increase with average kick magnitude initially $\left(\sigma=100,200 \mathrm{~km} \mathrm{~s}^{-1}\right)$, but becomes less and less sensitive with higher kicks $\left(\sigma=300,400 \mathrm{~km} \mathrm{~s}^{-1}\right)$. The basic reason for this behavior is that we are examining a certain subgroup of post-SN systems that satisfies two specific constraints: they are bound and have merger times shorter than $10^{10} \mathrm{yr}$. These two constraints act as a filter on the NS-NS population and all their properties: As $\sigma$ increases initially, the velocities of this subgroup increase as well. However, as kicks become higher, systems with even higher center-of-mass velocities no longer satisfy these two constraints and are "filtered out" of the population of interest to us. This behavior is in excellent agreement with the basic understanding of the effects of kicks on binary populations (e.g., Kalogera 1996). We note that the "Paczynski-like" distribution does not lead to significantly different results from the Maxwellian distributions, even though it includes a significant component of low-velocity kicks. The reason is again this "filtering" effect: formation of coalescing NS-NS requires some minimum kick (Fryer \& Kalogera 1997; Wex et al. 2000) and the existence of small kicks does not affect the properties of the final population 8 .

We should also mention that higher velocities are acquired by systems with more massive helium-star progenitors (we cover a range of $2-10 \mathrm{M}_{\odot}$ determined by the requirement that a NS is formed). However, such higher masses are disfavored by the mass function of progenitors (inverse power-law) and therefore their contribution in the final $Z$-distributions is limited. The range in $M_{0}$ is narrow enough that the details of the mass distribution become unimportant. This is clearly indicated by the insensitivity $(\lesssim 1 \%)$ of our results on the specific choice of the power-law index over a wide range of values $(1-3)$.

In Figure 2 a set of distribution functions (normalized to unity) of $T_{\mathrm{m}}$ are plotted, for different kick-magnitude distributions and for a specific helium-star mass. As in the case of velocities, the dependence of the distributions becomes much weaker as average kick magnitudes increase. It is interesting to note that the cases with typically higher $V_{\mathrm{CM}}$ also lead to typically shorter merger time scales. This correlation originates from the fact that velocities are higher for tighter binaries, which also experience faster orbital decay (and merging), and as a result, the expansion of the coalescing NS-NS population from their birth places is somewhat limited.

\footnotetext{
${ }^{8}$ The formation rate is of course greatly affected depending on the fraction of kick magnitudes that are in the "favored window" for the formation of tight NS-NS binaries. This sensitivity of formation is clearly evident in our results but does not affect our calculation of the scale height $Z_{0}$, which is independent of the overall rate. For this reason, we show only the normalized distributions of various parameters.
} 
Our final $Z$-distributions are shown in Figure 3. It is evident that - not surprisingly - the actual shape of these distributions is not a perfect exponential $\left(\exp \left(-|Z| / Z_{0}\right)\right.$ as assumed in studies of pulsar selection effects (this is true even if we plot the distributions as a function of $Z^{2}$, for the form $\exp \left(-Z^{2} / 2 Z_{0}^{2}\right)$. We explore the effect of this difference on the scale factors by adopting two different ways of obtaining a scale height for the NS-NS population. (i) For an exponential distribution, the fraction of the population with vertical distances smaller than $Z_{0}$ is equal to $63.2 \%$. For our calculated $Z$-distributions, we adopt a scale height so that $63.2 \%$ of the population lies at smaller vertical distances. For the four different kick-magnitude models, the derived scale heights lie in the range $0.8-1.7 \mathrm{kpc}$. Based on the $Z_{0}$ scaling derived by Curran \& Lorimer (1995), this $Z_{0}$-range leads to scale factors $\mathcal{S}$ in the ranges $45-60$ for PSR B1913+16 and 145 - 195 for PSR B1534+12. (ii) We note that although the derived $Z$-distributions cannot be well described by a single exponential, they can be fitted rather well by exponentials if they are divided into two segments. We choose the "break" point to be at $Z=0.5 \mathrm{kpc}$ and we calculate two scale heights: $0.3 \mathrm{kpc}$ for $Z<0.5 \mathrm{kpc}$ (same for all kick-velocity models) and $1.5-2.5 \mathrm{kpc}$ for $Z>0.5 \mathrm{kpc}$ (for the various kick-velocity models). We then use the scale heights and relative fractions of the two sub-populations and find the scale factors $\mathcal{S}$ in the ranges $46-61$ for PSR B1913+16 and $148-197$ for PSR B1534+12.

We find the agreement in the scale factors from these two different types of analysis quite encouraging. Accordingly, we adopt the following conservative ranges for the scale factors: $45-60$ for PSR B1913+16 and 145 - 200 for PSR B1534+12.

\subsection{NS-NS Lifetimes}

To obtain a coalescence rate for NS-NS binaries, estimates of their lifetimes are necessary. In particular, we are interested in the "observable" lifetimes of the systems, i.e., the time intervals during which the observed NS-NS binaries are detectable. They are the sum of the time since their formation (current age) and of their remaining lifetime.

The characteristic age $\tau_{c} \equiv P / 2 \dot{P}$, where $P$ and $\dot{P}$ are the spin period and its derivative, is often used as a measure of the current age of a radio pulsar. This is true assuming that magnetic dipole radiation roughly describes the pulsar emission, the magnetic braking index is equal to 3 , and the initial spin period was much smaller than the current period. It is not always clear that these assumptions are valid, but in many cases the characteristic age serves as a good approximation of the true pulsar age, especially in the absence of any other information. The characteristic ages for PSR B1913+16 and PSR B1534+12 are $1.1 \times 10^{8} \mathrm{yr}$ and $2.5 \times 10^{8} \mathrm{yr}$, respectively. However, the radio pulsars found in coalescing NS-NS binaries appear to have been recycled. This implies that the current spin periods are not much different than the initial periods at the end of the recycling phase. Arzoumanian et al. (1998) suggested that an alternative and possibly better way to obtain age estimates is to consider the spin history of these pulsars. They calculated the initial spin, $P_{0}$, as indicated by the intersection of the magnetic dipole spin-down 
line and the accretion spin-up line on the $P-\dot{P}$ pulsar diagram, and calculated the spin-down age

$$
T_{\mathrm{sd}}=\frac{P}{(n-1) \dot{P}}\left[1-\left(\frac{P_{0}}{P}\right)^{n-1}\right],
$$

where $n$ is the braking index. For $n=3$, they derive spin down ages of $65 \mathrm{Myr}$ and $200 \mathrm{Myr}$, for PSR B1913+16 and PSR B1534+12, respectively, lower than their characteristic ages by $40 \%$ and $20 \%$. These spin-down ages increase by a factor of 1.7 as $\mathrm{n}$ varies from 3 to 2 . Their sensitivity to the position of the spin-up line on the $P-\dot{P}$ plane is found to be much weaker. We consider these spin-down ages to be more reliable as they are better physically motivated. Further, for PSR B1913+16, kinematic constraints can be derived based on its position in the Galaxy and its measured transverse velocity. Wex et al. (2000) derived a minimum kinematic age of $35 \mathrm{Myr}$, for one intersection with the Galactic plane after its formation Arzoumanian et al. 1998 derived a higher value for the minimum kinematic age of $60 \mathrm{Myr}$, but this is the result of their simplifying assumption of a constant Galactocentric radius, which breaks down for ages longer than just a few Myr). This minimum kinematic age is consistent with both the characteristic and spin-down age of PSR B1913+16. Taking into account (i) the dependence of the spin-down ages on the braking index $n$ and on the position of the spin-up line, and (ii) the kinematic constraints for PSR B1913+16, we obtain conservative errors on $T_{\mathrm{sd}}$ : $65_{-30}^{+45} \mathrm{Myr}$, and $200_{-40}^{+140}$ Myr, for PSR B1913+16 and PSR B1534+12, respectively.

The remaining "observable" lifetimes of pulsars in coalescing NS-NS binaries are limited by a number of different factors. It is thought that pulsars cease to emit pulsed radiation as they cross the empirical "death line" on the $P-\dot{P}$ pulsar diagram $\left(\dot{P} P^{-5} \simeq 5 \times 10^{-17} \mathrm{~s}^{-5}\right.$; Ritchings 1976; Manchester \& Taylor 1977; Lyne \& Graham-Smith 1998). This line represents a constant magnetic field strength at the light cylinder and can be theoretically justified in the context of pulsar emission models in which the emission source is close to the light cylinder. The time to reach the death line sets a limit to the remaining observable lifetimes. However, for the recycled pulsars in these systems this "death-line" lifetimes are rather long $\left(2 \times 10^{10} \mathrm{yr}\right.$ and $6.7 \times 10^{9} \mathrm{yr}$ for PSR B1534+12 and PSR B1913+16, respectively) and do not lead to interesting constraints.

The merger event itself sets an upper limit to the lifetimes of the binaries. Merger times can be calculated accurately, given the measured binary characteristics and the rate of angular momentum loss due to gravitational radiation. They are $2.7 \times 10^{9} \mathrm{yr}$ and $3 \times 10^{8} \mathrm{yr}$ for PSR B1534+12 and PSR B1913+16, respectively and have been used in the studies of Narayan et al. (1991), Phinney (1991), and Curran \& Lorimer (1995). More recently, van den Heuvel \& Lorimer (1996) argued that the lifetime of the radio pulsars in the NS-NS are more strongly limited by luminosity evolution. They calculated the time at which the magnetic dipole energy loss rate (pulsar luminosity being proportional to this) drops below the level reached by a normal, young pulsar in its lifetime (assumed to be $10^{7} \mathrm{yr}$ ). The implicit assumption in this constraint is that the pulsar becomes undetectable when it becomes too faint. Although this is true given the flux limited pulsar surveys, it appears to be redundant since a correction for the faint end of the pulsar luminosity function, i.e., pulsars fainter than the minimum flux survey limits, is also applied to 
the birth rate estimate later.

Arzoumanian et al. (1998) have pointed out one last constraint imposed on the observable lifetime. It is related to the known selection effect of pulsar searches against pulsars in tight binaries. For tight orbits (orbital periods of a few hours) the Doppler shift of the pulse due to the rapidly changing acceleration of the pulsar leads to significant signal-to-noise reduction. The two systems of interest here could not have been detected with the completed surveys to date if their orbital periods were half their present values (Arzoumanian et al. 1998). PSR B1913+16 and PSR B1534+12 will have half the current orbital periods in $1.8 \times 10^{8} \mathrm{yr}$ and $2.2 \times 10^{9} \mathrm{yr}$, respectively.

We adopt the derived spin-down ages as the current ages of the two coalescing systems, and the times to reach half the current orbital period as their remaining lifetimes. These latter are by definition shorter than the merger times, although the difference between the two for the observed systems is quite small. The total lifetimes derived for PSR B1913+16 and PSR B1534+12 are $\simeq 2.5 \times 10^{8} \mathrm{yr}$ and $\simeq 2.5 \times 10^{9} \mathrm{yr}$, respectively. Note that the remaining lifetimes (times to reach half the current orbital periods) are considerably longer and much more accurate than the spin-down age estimates, and therefore the errors of the total lifetimes become rather small (less than 10\%). These longer remaining lifetimes also indicate that the two binary pulsars are still

relatively young. In fact, this is qualitatively expected based on considerations of pulsar luminosity evolution: it is easier to detected them early in their lifetime when they are still relatively bright. In $\S 3.5$ we present an analysis of the necessary corrections for luminosity evolution and faint pulsars and of the associated uncertainties.

\subsection{Beaming Fraction}

Detection of pulsars relies primarily on the pulsar beam of radiation intersecting the observer's line of sight. It has long been recognized that an upward correction must be applied to the empirical estimates of NS-NS coalescence rates to account for the fraction of pulsars that remain undetected not because of survey selection effects but because they do not beam in our direction (Narayan et al. 1991; Phinney 1991). This correction factor depends on the distribution of pulsar beam sizes which may be different for different types of pulsars (e.g., recycled or non-recycled). Studies of the pulsar emission geometry of mostly non-recycled pulsars have lead to the development of a number of spin period-dependent beaming models (e.g., Narayan \& Vivekanand 1983; Narayan (1987); Lyne \& Manchester 1988; Biggs 1990). The derived corrections for beaming fraction typically lie in the range $1-5$ and major uncertainties remain primarily because of our limited understanding of the pulsar emission process and geometry and because of limited data available for millisecond pulsars.

Previous studies of the NS-NS coalescence rate have not attempted to address this question in any detail and have either adopted a nominal value of 3 for the beaming correction factor or 
treated it as an unknown parameter.

We address this issue based on recent analyses of data on pulse profile-time evolution (with a long baseline) and polarization for PSR B1913+16 (Kramer 1998; Taylor 1999; Karastergiou et al. 2000; Weisberg \& Taylor 2000) and PSR B1534+12 (Arzoumanian et al. 1996; Stairs et al. 2000). It has now been confirmed by several groups that the PSR B1913+16 pulse profile is evolving with time at more than one frequency and that this time evolution can be successfully explained by pulsar precession due to misaligned spin and orbital angular momentum axes (for the origin and implications of this misalignment, see Wex et al. 2000). Weisberg \& Taylor (2000) analyzed a data set that includes recent high-accuracy data from the Arecibo observatory and found evidence for an elongated beam along the latitude direction with an elongation factor of $R=1.62$. The half-opening angle in the latitude direction is found to be $\rho=12^{\circ} .4$ and the magnetic inclination angle (relative to the spin axis) is $\alpha=156^{\circ}$. For PSR B1534+12 too, pulse-profile evolution has been confirmed, but the data are limited compared to PSR B1913+16 and do not allow a detailed analysis. A simpler analysis (using the rotating-vector model, see, e.g., Radhakrishnan \& Cooke 1969; Lyne \& Manchester 1988) gives a beam half-opening angle of $\rho=4^{\circ} .87$ and $\alpha=114^{\circ}$ (Arzoumanian et al. 1996). Future observations of PSR B1534+12 will extend the time baseline and could provide us with a better estimate of its beaming fraction.

Taking into account both pulsar beams, the beaming fraction is given by

$$
f_{b}=\left[\frac{2}{4 \pi} \int_{0}^{2 \pi} d \phi \int_{\alpha-\rho}^{\alpha+\rho} \sin \theta d \theta\right]^{-1} .
$$

We derive beaming fractions of 5.8 and 6.5, for PSR B1913+16 and PSR B1534+12, respectively. Based on these, we adopt an upward correction factor of 6 for pulsar beaming, and we apply it to the rate estimates we have obtained so far.

\subsection{Faint Pulsars and Small-Number Observed Sample}

Given that the estimates of the coalescence rate are based on the observed sample of coalescing NS-NS binaries and that pulsar surveys are flux-limited, it is necessary to include an upward correction to the rate estimate for the low-luminosity pulsars that are possibly not represented in the sample.

Curran \& Lorimer (1995) applied a simple correction (of a factor of 10) extrapolating an assumed pulsar luminosity function from the luminosity of the faintest object in the sample (PSR B1534+12) down to an assumed luminosity cut-off of $\sim 1 \mathrm{mJy} \mathrm{kpc}^{2}$. This correction probably represents an overestimate of the "missed" pulsars, since the detectability of faint pulsars depends on their flux (distance and luminosity), while the correction factor introduced by Curran $\&$ Lorimer (1995) implies that all pulsars fainter than the observed ones are non-detectable regardless of their position in the Galaxy. 
A more accurate correction factor was introduced by Arzoumanian et al. (1998), who convolved the detection volume integral with the luminosity function. However, with their method of obtaining a rate estimate they do not make use of any information on the observed sample or any weighting based on their Galactic distribution, and assume an average lifetime for the whole NS-NS population.

Here we present a statistical calculation of the "faint-pulsar" correction factor and its uncertainties. It applies to the basic method of calculating scale factors for each observed objects and is sensitive to the number of objects and how well these objects represent the luminosity function of the total Galactic population.

\subsubsection{Model}

We consider model pulsar populations (characterized by an assumed luminosity function) and a large number of model observed samples. Using a luminosity-dependent model for pulsar selection effects, we apply the method of scale factors to the model observed samples (as it is done with the real observed sample) and calculate the total estimated number of pulsars in the Galaxy, which is equal to the sum of the scale factors calculated for each pulsar in the observed sample. We obtain the correction factor for low-luminosity objects and the associated uncertainties based on a comparison between the estimated total number of pulsars and the assumed "true" population.

The population of both young radio pulsars and millisecond pulsars appears to be well described by a power-law luminosity function, $\phi(L)$, with a negative power-law index (Lyne et al. 1985; Cordes \& Chernoff 1997):

$$
\phi(L) \propto L^{-p} .
$$

Assuming a minimum luminosity, $L_{\min }$, for pulsars and that $p>1$ we normalize $\phi(L)$ to the total number of pulsars in the Galaxy, $N_{G}$ :

$$
\phi(L)=(p-1) L_{\min }^{p-1} N_{G} L^{-p}, \quad L \geq L_{\min },
$$

where we have assumed that the distribution extends to very large luminosities. The normalization of $\phi(L)$ and in general the statistics of the population are insensitive to the exact value of the maximum luminosity. Cordes \& Chernoff (1997) presented a maximum likelyhood analysis of the properties of millisecond pulsars and obtained best-fit values for $p$ and $L_{\min }: p=2 \pm 0.2$ and $L_{\text {min }}=1.1_{-0.5}^{+0.4} \mathrm{mJy} \mathrm{kpc}^{2}$ (for non-recycled pulsars, it is $p \gtrsim 1$; see Lyne et al. 1985; Lorimer et al. 1993). For our standard case, we use $p=2$ and $L_{\text {min }}=1 \mathrm{mJy} \mathrm{kpc}^{2}$. In our parameter study we examine the sensitivity of the results to these values.

The scale factors, $\mathcal{S}$, as a function of spin period and luminosity, have been calculated in detailed studies of pulsar populations and selection effects (e.g., Narayan 1987). The dependence on luminosity appears to be well described by three segments: (i) At high luminosities pulsars can be detected essentially anywhere in the Galaxy, so $\mathcal{S}(L)=1$; (ii) At intermediate luminosities 
detection is volume-limited in a disk-like population, so $\mathcal{S}(L) \propto L^{-1}$; (iii) At sufficiently low luminosities the limiting distance from the Sun is smaller than the disk height and detection is volume limited in a homogeneous population, so $\mathcal{S}(L) \propto L^{-3 / 2}$. By matching the segments at the luminosity boundaries we obtain

$$
\begin{aligned}
\mathcal{S}(L) & =L_{1}^{1 / 2} L_{2} L^{-3 / 2} & L_{\min }<L<L_{1} \\
& =L_{2} L^{-1} & L_{1}<L<L_{2} \\
& =1 & L_{2}<L
\end{aligned}
$$

Guided by the results of Narayan (1987) we choose, for our standard case, $L_{1}=30 \mathrm{mJy} \mathrm{kpc}^{2}$ and $L_{2}=3000 \mathrm{mJy} \mathrm{kpc}^{2}$. In what follows we ignore the dependence of $\mathcal{S}(L)$ on pulsar spin period. This simplification is probably well justified, since the pulsars found in NS-NS binaries cover a relatively narrow range of spin periods $(40-60 \mathrm{~ms})$ and the variation of $\mathcal{S}(L)$ with period is rather small (Narayan 1987).

The luminosity function of observed pulsars, $N(L)$, is then given by $N(L)=\phi(L) / \mathcal{S}(L)$ and we can calculate the mean number of observed pulsars as a function of $L_{\min }, L_{1}, L_{2}$, and $p$, using equations (2) and (3):

$$
\begin{aligned}
<N_{\text {obs }}>= & \int_{L_{\min }}^{\infty} N(L) d L \\
= & N_{G}\left[(p-1)\left(p-\frac{5}{2}\right)^{-1} L_{2}^{-1}\left(L_{\min }^{3 / 2} L_{1}^{-1 / 2}-L_{\min }^{p-1} L_{2}^{2-p}\right)\right. \\
& \left.+\frac{p-1}{p-2} L_{\min }^{p-1} L_{2}^{-1}\left(L_{1}^{2-p}-L_{2}^{2-p}\right)+L_{\min }^{p-1} L_{2}^{1-p}\right] .
\end{aligned}
$$

The case of $p=2$ is singular for the above equation. For $p=2,<N_{\mathrm{obs}}>$ is given by

$$
<N_{\mathrm{obs}}>=N_{G}\left[2 L_{2}^{-1}\left(L_{\min }-L_{\min }^{3 / 2} L_{1}^{-1 / 2}\right)+L_{\min } L_{2}^{-1} \ln \left(L_{2} / L_{1}\right)+L_{\min } L_{2}^{-1}\right],
$$

which for our the choice of $L_{\min }, L_{1}, L_{2}$ in our standard case becomes: $\left\langle N_{\text {obs }}>\simeq N_{G} / 415\right.$.

It can be shown that, in this simple (proof-of-principle) model, the mean estimated total number of pulsars is actually equal to the true total number of pulsars in the Galaxy. The mean scale factor for the observed population is

$$
<\mathcal{S}(L)>=\frac{\int_{L_{\min }}^{\infty} \mathcal{S}(L) N(L) d L}{\int_{L_{\min }}^{\infty} N(L) d L}=\frac{\int_{L_{\min }}^{\infty} \phi(L) d L}{<N_{\mathrm{obs}}>}=\frac{N_{G}}{<N_{\mathrm{obs}}>} .
$$

In the case of an observed sample, the mean scale factor can be expressed in a discrete form:

$$
<\mathcal{S}(L)>=\frac{\sum_{i} \mathcal{S}_{i}\left(L_{i}\right)}{N_{\mathrm{obs}}}=\frac{N_{\mathrm{est}}}{N_{\mathrm{obs}}}
$$

From equations (18) and (20), we obtain $N_{\text {est }}=\frac{N_{G}}{<N_{\text {obs }}>} N_{\text {obs }}$ and its mean value then is $<N_{\text {est }}>=N_{G}$. We confirm this equality in our numerical results described in the next section (see also equation [A8] in the Appendix). 


\subsubsection{Calculations and Results}

We use the above model for the pulsar properties and selection effects and perform Monte Carlo simulations to investigate the "faint-pulsar" correction to the rate estimate and its dependence on the number of objects in the observed sample.

For a given value of the mean number of observed pulsars, $\left\langle N_{\text {obs }}\right\rangle$, we take $N_{\text {obs }}$ to be a random number drawn from a Poisson distribution with a mean equal to $\left\langle N_{\text {obs }}\right\rangle$. We generate a large number of model observed samples consisting of $N_{\text {obs }}$ objects. For each object in the sample, we choose a luminosity $L_{i}$ drawn from the observed luminosity function, $N(L)$. We then evaluate the individual scale factors, $\mathcal{S}_{i}\left(L_{i}\right)$, and the estimated total number of pulsars in the Galaxy:

$$
N_{\text {est }}=\sum_{i=1}^{N_{\text {obs }}} \mathcal{S}_{i}\left(L_{i}\right) .
$$

For a given value of $\left\langle N_{\text {obs }}\right\rangle$, we repeat the calculation for a large number $\left(10^{5}\right)$ of values of $N_{\text {obs }}$, and we obtain the distribution of $N_{\text {est }}$ as a function of $\left\langle N_{\text {obs }}\right\rangle$. To characterize this distribution we use its mean, median, first quartile (25\%) and third quartile $(75 \%)$.

The model estimated total number $N_{\text {est }}$ (eq. 20) is obtained in the same way as it is done for the real observed sample. The difference is that, in the model calculations, we can actually compare the distribution of $N_{\text {est }}$ to the "true" total number of pulsars $N_{G}$ as a function of $<N_{\text {obs }}>$. Our results for our standard case $\left(p=2, L_{\min }=1 \mathrm{mJy} \mathrm{kpc}^{2}, L_{1}=30 \mathrm{mJy} \mathrm{kpc}^{2}\right.$, and $L_{2}=3000 \mathrm{mJy} \mathrm{kpc}^{2}$ ) are shown in Figure 4. As shown in $\S 3.5 .1$, the mean of the distribution is equal to the "true" total number of pulsars in the Galaxy. For large values of $\left\langle N_{\text {obs }}\right\rangle$ the distribution of $N_{\text {est }}$ is narrow and its median follows closely its mean value, an indication that the method of estimated the total pulsar number using the scale factors leads to reliable results. On the contrary, for small values of $\left\langle N_{\text {obs }}>(\lesssim 10)\right.$, the distribution becomes wider and the median deviates systematically to values lower than the total Galactic number of pulsars (the distribution becomes highly skewed with an extended tail to large values). For very low $\left\langle N_{\text {obs }}\right\rangle(\lesssim 3)$, the mean is dominated by a small number of realizations for the observed sample, since in more than $75 \%$ of the cases $N_{\text {est }}$ lies below the mean. For $<N_{\text {obs }}>=2$, the derived $N_{\text {est }}$ could be an underestimate of the true number by up to 2 orders of magnitude.

The $N_{\text {est }}$-distributions are shown in Figure 5 (in $N_{\text {est }} f\left(N_{\text {est }}\right)$ form), for three different choices of $\left\langle N_{\text {obs }}\right\rangle=2,10,50$. The high degree of skewness towards low values of $N_{\text {est }}$ for a small-number sample is evident. The distributions becomes much more narrowly concentrated around their almost equal mean and median values for large-number samples. In the appendix we derive analytic expressions for the moments of the distribution along with its relative variance and skewness.

The reason for the extreme skewness of the probability distribution of the estimated number of pulsars is primarily connected to the properties of their luminosity function. The true population is dominated by faint pulsars that are hard to detect in pulsar searches. When only a small 
number of pulsars is drawn out of such a population, it is more probable that they will be bright pulsars. Therefore a small-number sample is more likely dominated by bright pulsars. This leads to large values of the estimated volume out to which they could be detected, consequently to low values of their scale factors, and hence their sum, $N_{\text {est }}$, represents an underestimate of the true total number.

It becomes evident that a upward correction factor must be applied to the estimated rate to account for this effect. We calculate the correction based on the ratio of the "true" total number of objects to the estimated total number, in our model. The reasons for this correction factor are actually equivalent to those of the corrections for low-luminosity pulsars, adopted by authors in previous studies: for example, an upward correction of a factor of 10 was introduced by Curran \& Lorimer (1995) based on a simple extrapolation of the luminosity function down to a minimum luminosity. The analysis we present here shows that this extension to low luminosities is actually quite uncertain (for reasons other than just the uncertainties in the values of $p, L_{\min }, L_{1}$, and $L_{2}$ ). The uncertainty rapidly increases for small-number samples and can be as large as two orders of magnitude for an observed sample of only two objects.

The above results are quite robust and general as long as the luminosity function is dominated by faint pulsars and the detection process is such that faint objects are harder to detect, i.e., flux-limited surveys. The quantitative details of the degree of underestimation of the true total pulsar number turn out to be somewhat sensitive to the values of the input parameters, $p, L_{\min }$, $L_{1}$, and $L_{2}$. We have run simulations for a set of different values for all these parameters ( $p$ : $\left.1.8,2 ; L_{\min }: 1,3 ; L_{1}: 10,30,100 ; L_{2}: 1000,3000\right)$ guided by the results of Cordes \& Chernoff (1997) and Narayan (1987). From our parameter study, we have eliminated cases (sets of $p, L_{\min }$, $L_{1}$, and $L_{2}$ values) in which the normalization between $N_{G}$ and $\left\langle N_{\text {obs }}>\right.$ deviates from current expectations, i.e., $N_{G} /<N_{\text {obs }}>\sim 100$, by a factor larger than $\sim 5$. This constraint originates in the (order-of-magnitude) estimates of the total number of pulsars in the galaxy $\left(\sim 10^{5}\right)$ and the number of observed pulsars $\left(\sim 10^{3}\right)$. For all the cases that satisfy this constraint, our results for the median, first, and third quartile of the $N_{\text {est }}$-distributions are given in Table 1 (see also Figure 6 ). We find that, for all models, a minimum number of 5-10 observed objects ( 7 for our standard case) is necessary to obtain a median that underestimates the true number by a factor smaller than 3. At $<N_{\text {obs }}>=2$ the true total pulsar number is underestimated typically by factors of $\lesssim 2$ up to $\simeq 300(\simeq 2-200$ for our standard case with the median at $\simeq 15)$.

\subsubsection{Effect of Distance Errors}

So far we have neglected any uncertainties in the distance estimates of the pulsars. Pulsar distances are inferred based on their dispersion measure and an electron-density model (e.g., Taylor \& Cordes 1993) and are known to be uncertain. Here we examine their effect on the "faint-pulsar" correction factors derived in $\S 3.5 .2$. 
We modify the Monte Carlo simulations described above to include distance errors. For each object in the model samples, we generate not only a luminosity $L$ but also a position in the Galaxy described by a galactocentric radius $R$, a vertical distance from the Galactic plane $Z$, and an azimuthal angle $\Phi$. These three parameters are assigned to the observed objects according to the probability distribution $F_{p}$

$$
F_{p}(R, Z, \Phi) \propto \frac{1}{2 \pi} \exp \left(-\frac{R^{2}}{2 R_{0}^{2}}-\frac{|z|}{z_{0}}\right),
$$

where $R_{0}=4 \mathrm{kpc}$ and $z_{0}=1 \mathrm{kpc}$ are the radial and vertical scale lengths. Given the position of the pulsar, we calculate its "true" distance $D$ from the Sun. We then generate in our Monte Carlo simulations an "inferred" distance $D^{\prime}$. This distance is drawn from a Gaussian distribution with a mean equal to $D$ and a standard deviation $\sigma=f D$, where $f$ is a factor smaller than unity: $f\left(D^{\prime}\right) \propto \exp \left[-\left(D^{\prime}-D\right)^{2} /\left(2(f D)^{2}\right)\right]$. We use the two distances and the true luminosity and assign an "observed" luminosity $L^{\prime}=L\left(D^{\prime} / D\right)^{2}$ to each of the Monte Carlo "observed" pulsars. Scale factors (eq. [16]) are calculated using $L^{\prime}$ and from them we obtain $N_{\text {est }}$ (eq. [20]).

We obtain results on the distribution of $N_{\text {est }}$ compared to the model "true" total number of pulsars in the Galaxy for the case of $20 \%$ distance errors $(f=0.2)$ and the extreme case of $80 \%$ errors $(f=0.8)$ \% . We find that errors at the $20 \%$ level are too small $(\lesssim 1 \%)$ to have any

effect on the $N_{\text {est }}$-distributions. The effect starts becoming non-negligible at $f \gtrsim 0.5$ and is rather significant (factor of 2-3) for $f=0.8$. For such large $f$-values, the distribution of $N_{\text {est }}$ becomes broader leading ultimately to highly uncertain empirical rate estimates. However, we do not expect that such large distance errors are realistic, for the general pulsar population. Distance estimates based on the Taylor \& Cordes (1993) electron density model have been found to have typical errors of 20-30\% and therefore we conclude the "faint-pulsar" correction factors should not be increased, because of pulsar distance errors.

\subsection{Conclusions on the Galactic Coalescence Rate}

We use the derived scale factors and lifetimes for the two coalescing systems, to obtain an estimate of the Galactic coalescence rate, which we can further correct for beaming and the faint end of the pulsar luminosity function. We also identify the most dominant sources of the associated uncertainty.

Based on our results on the NS-NS scale height we obtained $\mathcal{S}_{1913+16}=45-60$ and $\mathcal{S}_{1534+12}=145-200$. We combine these results with the estimated lifetimes of the two systems and obtain an estimate for the NS-NS coalescence rate in the range $2-4 \times 10^{-7} \mathrm{yr}^{-1}$.

\footnotetext{
${ }^{9}$ We have explored the dependence of our results on $R_{0}$ and $z_{0}$ by running models for $R_{0}=8 \mathrm{kpc}$ and $z_{0}=3 \mathrm{kpc}$ and the changes are negligible.
} 
This Galactic rate must be further corrected for the fraction of coalescing NS-NS binaries with pulsars that do not beam in our direction. We found this upward correction factor to be $\simeq 6$ leading to rates in the range $(1-2.5) \times 10^{-6} \mathrm{yr}^{-1}$. We further have to correct the rate for the fraction of pulsars at the faint end of the luminosity function that are hard to detect and are not represented in the observed sample. For low-number observed samples $(\lesssim 5-10)$, in particular, this "faint-pulsar" correction factor has a broad distribution and typically contributes to an upward revision of the rate. The correction factors are found to be as high as $\sim 200$ (see Table 1). As a result we estimate the Galactic NS-NS coalescence rate to lie in the range $\simeq 10^{-6}-5 \times 10^{-4} \mathrm{yr}^{-1}$, where the extent of this range is primarily dominated by the uncertainty in the "faint-pulsar" correction for a small-number sample.

In our analysis we have obtained estimates and the associated uncertainties for each of the relevant factors, separately. Therefore, it will be straightforward to update our coalescence rate estimate, if new and improved estimates of $\mathcal{S}_{1913+16}$ and $\mathcal{S}_{1534+12}$ are obtained in the future.

\section{EXTRAGALACTIC RATE EXTRAPOLATION}

Interferometric ground-based gravitational-wave observatories currently under construction are expected to be sensitive to NS-NS inspiral events out to extragalactic distances. Specifically for LIGO, the most up-to-date estimates of the maximum detection distances place them at $\simeq 20 \mathrm{Mpc}$ and $\simeq 350 \mathrm{Mpc}$, for LIGO I and II, respectively (Gustafson et al. 1999; Finn 2000, private communication). Hence, we need to extrapolate the Galactic coalescence rate estimated in $\S 3$ out to the volume of the Universe accessible to LIGO I and II. There are several ways of performing this extrapolation, using, for example, scalings with galaxy mass, luminosity, or star formation rate. The implicit assumption in such types of scalings is that (1) the NS-NS inspiral rate is proportional to the formation rate of massive stars, (2) the properties (mass function, binary fraction, etc.) of the primordial binary population in galaxies (within distances of interest) are not grossly different than those of the Galactic population, and (3) the star formation history out to these distances has been roughly uniform, i.e., the fractions of starburst galaxies and ellipticals are rather small.

Phinney (1991) extrapolated the Galactic inspiral rate based on estimates of the B-band luminosity density of the universe obtained from galaxy counts (Efstathiou et al. 1988) and the

B-band luminosity of the Milky Way $\left(\simeq 2 \times 10^{10} \mathrm{~L}_{\odot, B}\right)$. He also included an upward correction, for any reprocessed B-band luminosity that is emitted in the infrared, and a downward correction, for the contribution of E,S0 galaxies to the B-band luminosity, since E,S0 galaxies stopped forming stars billions of years ago and are not likely to produce very many inspiral events at present. Narayan et al. (1991) obtained a different extrapolated estimate based on the average galaxy number density out to a few hundred Mpc, whereas Curran \& Lorimer (1995) used the number density of galaxy clusters, number of galaxies per cluster, and average size of voids between superclusters. The last two approaches include the additional assumption that each galaxy out to 
the distances of interest has the same NS-NS inspiral rate as the Milky Way.

In the present study we adopt a method of extrapolation similar to that used by Phinney (1991), but with up-to-date estimates of the B-band and infrared (IR) luminosity densities of the nearby universe and of the Milky Way B-band luminosity.

Luminosity functions have been derived for the most recent, completed, and relatively large-volume galaxy surveys both in the blue, the Stromlo-APM Redshift Survey with a sample median redshift of 0.05 (Loveday et al. 1992), and in the red, the Las Campanas Redshift Survey with a sample median redshift of 0.1 (Lin et al. 1996). Although the details of the two luminosity functions (after corrections for the difference in colors) are different, the integrated luminosity densities are found to be very similar, equal to $\simeq(1.4 \pm 0.1) \times 10^{8} h \mathrm{~L}_{\odot} \mathrm{Mpc}^{-3}$ $\left(h \equiv H_{0} / 100 \mathrm{~km} \mathrm{~s}^{-1} \mathrm{Mpc}^{-1}\right)$. This result is also consistent with preliminary results from the Sloan Digital Sky Survey Blanton et al. 2000). In contrast to Phinney (1991), we do not include a downward correction for the fraction of E,S0 galaxies because it is balanced by a similar correction of the Galactic B-band luminosity for the contribution from the bulge. However, following Phinney (1991), we do apply a correction for the fraction of the infrared luminosity density due to re-processed blue light by dust. We use the far-infrared $(40-100 \mu \mathrm{m})$ luminosity density derived by Saunders et al. (1990) for a sample of IRAS galaxies, and we further increase it by $30 \%$ to account for emission down to $12 \mu \mathrm{m}$ (see Soifer \& Neugebauer 1991): $L_{\mathrm{IR}} \simeq(3.6 \pm 0.4) \times 10^{7} h \mathrm{~L}_{\odot} \mathrm{Mpc}^{-3}$. We also adopt results of spectral syntheses (following Phinney 1991) showing that in the absence of any extinction half of this infrared light comes from reprocessed blue light. Then, depending on the assumed extinction law, we obtain an "intrinsic" B-band luminosity density in the range: $1.5-2 \times 10^{8} h \mathrm{~L}_{\odot} \mathrm{Mpc}^{-3}$. Note that the statistical uncertainties of the luminosity densities are overshadowed by the uncertainties in the dust extinction law.

For the B-band luminosity of our Galaxy, we obtain an estimate using the B-band Tully-Fisher relation from Yasuda et al. (1997) (their equation [5]) and adopting a circular velocity at the solar radius equal to $220 \mathrm{~km} \mathrm{~s}^{-1}$ (Feast \& Whitelock 1997). This circular velocity is also consistent with the Galactic potential used in $\S 3.2$, which gives a velocity of $222 \mathrm{~km} \mathrm{~s}^{-1}$ at $8 \mathrm{kpc}$ from the Galactic center). We calculate the blue absolute magnitude of the Milky Way to be -20.11 , which implies that the Galactic B-band luminosity equal to $9 \times 10^{9} \mathrm{~L}_{\odot}$. This value is also consistent with that of NGC 891, a galaxy very similar to the Milky Way with the same circular velocity. Our estimate of the blue luminosity of the Galaxy is about a factor of 2 lower than the one used by Phinney (1991). His adopted value is taken from a study by van der Kruit (1987), where a rather large value of the disk radial scale length was assumed ( $5 \mathrm{kpc})$. Studies of the Galactic disk in the IR lead to scale length estimates in the range $2.3-2.6 \mathrm{kpc}$ (Freudenreich 1998; Drimmel \& Spergel 2000), whereas corresponding estimates in the blue lead to values higher by about 30\% de Jong \& van der Kruit 1994). These low values are also consistent with studies of disk kinematics (see Dehnen \& Binney 1998). Since the total luminosity scales roughly with the square of the scale length, these lower estimates are consistent with our lower (by a factor of 2) estimate of the blue luminosity of the Milky Way. 
Based on the above estimates and corrections, we derive the scaling factor for the extragalactic extrapolation of the Galactic rate, i.e., the ratio of the B-band luminosity density to the B-band luminosity of the Milky Way in the range: $(1-1.5) \times 10^{-2} \mathrm{Mpc}^{-3}$, for $h=0.65$ (the effective maximum distances of reach for LIGO I and II have been calculated for $h=0.65$ ).

In principle, another way of obtaining an extrapolation factor is to use a scaling based on star formation rate estimates. However, at present these are much more uncertain than the results obtained from luminosity densities. For comparison, we derive a scaling factor using (i) a Galactic

star formation rate of $1-3 \mathrm{M}_{\odot} \mathrm{yr}^{-1}$, which includes recycling of molecular gas (Blitz 1997; Lacey \& Fall 1985), and (ii) a star formation density in the local Universe of $1.2-4 \times 10^{-2} \mathrm{M}_{\odot} \mathrm{yr}^{-1} \mathrm{Mpc}^{-3}$ (for $h=0.65$; Madau, et al. 1998; Sullivan et al. 2000). Consequently, we obtain a highly uncertain scaling factor to lie in the range $0.4-4 \times 10^{-2} \mathrm{Mpc}^{-3}$.

In what follows, we adopt the scaling for the extragalactic extrapolation based on the B-band luminosities.

\section{DISCUSSION}

We use the observed sample of close NS-NS binaries that will coalesce in less than $10^{10} \mathrm{yr}$ to estimate the Galactic NS-NS coalescence. We study in detail the uncertainties involved in this estimate and we find that the coalescence rate lies in the range: $10^{-6}-5 \times 10^{-4} \mathrm{yr}^{-1}$. We also calculate a simple scaling law for the extrapolation of this rate to extragalactic distances, based on the B-band luminosities of galaxies. These combined with the maximum detection distances that are expected to be reachable with LIGO $(20 \mathrm{Mpc}$ and $350 \mathrm{Mpc}$ for LIGO I and II, respectively, calculated for $h=0.65$; Finn 2000, private communication), lead us to estimates of the NS-NS inspiral detection rates, for LIGO I and II, in the ranges $3 \times 10^{-4}-2.5 \times 10^{-1} \mathrm{yr}^{-1}$ and $2-1300 \mathrm{yr}^{-1}$, respectively. We conclude that the prospects for detection of such inspiral events by LIGO II are strongly encouraging.

In this study we have given special attention to the various uncertainties associated with these empirical rate estimates and tried to quantify them wherever possible. We find that, by a very large margin, the most dominant source of uncertainty is the necessary correction for undetectable pulsars at the faint end of the luminosity function (see $\S 3.5$ ). Because of the small number of objects in the observed sample, this correction factor covers a wide range of values and can be as high as $\sim 200$. Uncertainties originating from the dynamical evolution of the radio pulsar population and pulsar lifetimes are within a factor of 2 , whereas uncertainties in the beaming fraction and the extragalactic extrapolation are found to be less important $(\lesssim 50 \%)$.

It is evident that any expectations for reducing this two-orders-of-magnitude uncertainty in the empirical coalescence rate strongly depend on the discovery of more close NS-NS binaries. We have shown that if the sample increases to $\sim 10$ objects then the correction for the faint end of the radio luminosity function can be reduced to just a factor of a few or less (see Figures 4 \& 
6). The ongoing Parkes Multibeam Survey (Lyne et al. 2000) could possibly contribute to the increase of the sample of coalescing NS-NS systems. PSR J1141-6545 was initially considered as a candidate NS-NS but follow-up measurements of relativistic parameters imply a total mass for the binary system low enough that the hypothesis of a NS-WD binary instead of a NS-NS binary appears to be more probable (Kaspi et al. 2000). We note that, based on the characteristic age of this pulsar (1.4 Myr) and the time to reach the pulsar "death-line" (29 Myr), were this system a NS-NS binary, its contribution to the coalescence rate would be $\mathcal{S} \times 3.3 \times 10^{-8} \mathrm{yr}^{-1}$, where $\mathcal{S}$ would be its scale factor. To our knowledge, only about half of the complete Parkes Multibeam Survey data set has been analyzed (although more extensive acceleration searches will follow), so it is reasonable to expect that new NS-NS binaries could be discovered in the next few years.

We note that the rate estimates we derive here are relevant to NS-NS binaries with recycled, hence long-lived radio pulsars, like the ones detected in the observed systems. Recent results of theoretical calculations of NS-NS formation indicate that systems can form without any of the two NS having a chance to be recycled by accretion (Belczynski \& Kalogera 2001), and that a significant population of non-recycled NS-NS binaries with very short radio-pulsar lifetimes can exist in the Galaxy. The possible existence of such a population implies an increase of the NS-NS coalescence rate by small but non-zero factors according to the results in Belczynski \& Kalogera (2001).

Apart from actual estimates of the coalescence rates, observed samples of radio pulsars have also been used to derive upper limits to the Galactic coalescence rate. Based on the absence of young radio pulsars found in NS-NS binaries, Bailes (1996) derived an upper limit of $\sim 10^{-5} \mathrm{yr}^{-1}$. Arzoumanian et al. (1998) raised this limit to $\sim 10^{-4} \mathrm{yr}^{-1}$ using a more detailed analysis of the same basic argument. These limits were calculated prior to the study by Belczynski \& Kalogera (2001) and they are expected to increase in view of them. Kalogera \& Lorimer (2000) derived a similar upper limit $\left(\sim 10^{-4} \mathrm{yr}^{-1}\right)$, although based on completely different considerations, related to the formation branching ratios of radio pulsars like those in close NS-NS binaries and their possible single counterparts. It is quite encouraging to see that both limits are in agreement with each other and the range of coalescence rates we obtained here is also consistent (within a factor of 5) with these upper limits. If we adopt these upper limits strictly then the LIGO II detection rate is reduced to about $2-300$ events per year.

We can also compare our results to the theoretical estimates for the NS-NS coalescence rates obtained from studies of the evolution of binary populations until the formation of close NS-NS systems (e.g., Lipunov et al. 1997; Fryer et al. 1998; Portegies-Zwart \& Yungel'son 1998; Bethe \& Brown 1998; Fryer et al. 1999; Bulik et al. 1999; Grishchuk et al. 2001). The results of these studies have been summarized elsewhere (e.g., Kalogera 2000a; Kalogera \& Lorimer 2000) and the rates have been found to cover a wide range of 3-4 orders of magnitude. The dominant uncertainties in these studies are related to multiple supernovae and NS kicks, the treatment of dynamically unstable mass-transfer episodes, and the properties of the primordial binary populations (e.g., mass ratios). The accuracy of these purely theoretical estimates could be improved, if a set of 
observational constraints (e.g., different supernova rates, statistics of Wolf-Rayet populations) are imposed on the models and their absolute normalization (see Belczynski, Kalogera, \& Bulik 2000). It is evident that, at present, studies of the observed sample of NS-NS binary pulsars provide us with a more accurate estimate of the NS-NS coalescence rate.

VK would like to thank T. Abel, T. di Matteo, C. Kochanek, M. Kramer, D. Lorimer, C. Metzler, and I. Stairs for helpful and stimulating discussions. This work was supported in part by the Smithsonian Astrophysical Observatory in the form of a Harvard-Smithsonian Center for Astrophysics Postdoctoral Fellowship and a Clay Fellowship to VK, and in part by NSF grants PHY 9507695 to RN and AST 96-18357 to JHT.

\section{A. Moments of the Distribution of Estimated Pulsar Numbers}

The estimated number of pulsars $N_{\text {est }}$ (see eq. [22]) can be written as

$$
N_{\mathrm{est}}=\sum_{i} N_{i}=\sum_{i} n_{i} s_{i}
$$

where $\left\langle n_{i}\right\rangle=\frac{\phi\left(L_{i}\right)}{\mathcal{S}\left(L_{i}\right)} \Delta L$ and $\Delta L$ is the width of luminosity bins. We denote the probability distribution of $N_{\text {est }}$ with $p\left(N_{\text {est }}\right)$ and that of $N_{i}$ with $p_{i}\left(N_{i}\right)$. If $\chi(t)$ and $\chi_{i}(t)$ are the Fourier transforms of $p\left(N_{\text {est }}\right)$ and $p_{i}\left(N_{i}\right)$, respectively, then the Fourier convolution theorem implies that

$$
\chi(t)=\prod_{i} \chi_{i}(t)
$$

We use the characteristic function of a Poisson process for $N_{i}$ and therefore:

$$
\chi_{i}(t)=\exp \left\{\frac{\phi\left(L_{i}\right)}{\mathcal{S}\left(L_{i}\right)} \Delta L\left[\exp \left(i \mathcal{S}\left(L_{i}\right) t\right)-1\right]\right\} .
$$

Equations (A2) and (A1) imply that

$$
\begin{aligned}
\log \chi(t) & =\sum_{i} \log \chi_{i}(t) \\
& =\sum_{i} \frac{\phi\left(L_{i}\right)}{\mathcal{S}\left(L_{i}\right)} \Delta L\left[\exp \left(i \mathcal{S}\left(L_{i}\right) t\right)-1\right] \\
& =\int d L \frac{\phi\left(L_{i}\right)}{\mathcal{S}\left(L_{i}\right)}\left[\exp \left(i \mathcal{S}\left(L_{i}\right) t\right)-1\right]
\end{aligned}
$$

We can then use the moment equation

$$
\mu^{r}=\frac{d^{r} \chi}{\left.d(i t)^{r}\right|_{t=0}}
$$

\footnotetext{
${ }^{10}$ In what follows we use the notation from $\S 3.5$
} 
to compute then mean, root-mean-square, and skewness of the distribution

$$
\begin{gathered}
\left\langle N_{\text {est }}\right\rangle=\int d L \phi(L)=N_{G}, \\
\left\langle N_{\text {est }}^{2}\right\rangle=\left\langle N_{\text {est }}\right\rangle^{2}+\int d L \phi(L) \mathcal{S}(L), \\
\left\langle N_{\text {est }}^{3}\right\rangle=\left\langle N_{\text {est }}\right\rangle^{3}+3\left\langle N_{\text {est }}\right\rangle \int d L \phi(L) \mathcal{S}(L)+\int d L \phi(L) s^{2}(L) \\
=3\left\langle N_{\text {est }}\right\rangle\left\langle N_{\text {est }}^{2}\right\rangle-2\left\langle N_{\text {est }}\right\rangle^{3}+\int d L \phi(L) s^{2}(L) .
\end{gathered}
$$

The above expression can be used to calculate the magnitude of dispersion of $N_{\text {est }}$ relative to its expected value and the skewness of the $N_{\text {est }}$-distribution relative to its dispersion.

Ultimately we are interested in the magnitude of the variance and the skewness and their dependence on $\left\langle N_{\text {obs }}>\right.$. As it can be seen from its definition in $\S 3.5, \mathcal{S}(L)$ does not depend on $<N_{\text {obs }}>$, but instead depends only on $p, L_{\text {min }}, L_{1}$, and $L_{2}$ (and $L$ of course). On the other hand, $\phi(L)$ is proportional to $N_{G}$, and hence proportional to $\left\langle N_{\text {obs }}>\right.$ (see eqs. [16], [18]). Also the integrals in eqs. (A8)-(A10) are independent of $\left\langle N_{\text {obs }}>\right.$. A measure of the relative dispersion is given by

$$
\frac{\left[\int\left(N_{\text {est }}-\left\langle N_{\text {est }}\right\rangle\right)^{2} f\left(N_{\text {est }}\right) d N_{\text {est }}\right]^{1 / 2}}{\left\langle N_{\text {est }}\right\rangle}
$$

which is equal to

$$
\frac{\left[\left\langle N_{\mathrm{est}}^{2}\right\rangle-\left\langle N_{\mathrm{est}}\right\rangle^{2}\right]^{1 / 2}}{\left\langle N_{\mathrm{est}}\right\rangle}=\frac{\left[\int d L \phi(L) \mathcal{S}(L)\right]^{1 / 2}}{\int d L \phi(L)} \propto\left\langle N_{\mathrm{obs}}\right\rangle^{-1 / 2} .
$$

A measure of the skewness of the $N_{\text {est }}$-distribution relative to its dispersion is given by

$$
\frac{\int\left(N_{\text {est }}-\left\langle N_{\text {est }}\right\rangle\right)^{3} f\left(N_{\text {est }}\right) d N_{\text {est }}}{\left[\left\langle N_{\text {est }}^{2}\right\rangle-\left\langle N_{\text {est }}\right\rangle^{2}\right]^{3 / 2}}
$$

which is equal to

$$
\frac{\left\langle N_{\mathrm{est}}^{3}\right\rangle-3\left\langle N_{\mathrm{est}}\right\rangle\left\langle N_{\mathrm{est}}^{2}\right\rangle+2\left\langle N_{\mathrm{est}}\right\rangle^{3}}{\left[\int d L \phi(L) \mathcal{S}(L)\right]^{3 / 2}}=\frac{\int d L \phi(L) s^{2}(L)}{\left[\int d L \phi(L) \mathcal{S}(L)\right]^{3 / 2}} \propto\left\langle N_{\mathrm{obs}}\right\rangle^{-1 / 2} .
$$

It is evident that the relative variance and skewness of the distribution decrease as the number of systems in the observed sample increase.

The proportionality constants in equations (A12) and (A14) provide us with information about the magnitude of the variance relative to the mean and the skewness relative to the variance. These constants can be calculated analytically using equations (16) - (18). As expected based on our discussion in $\S 3.5$, we find that both the variance and the skewness are greatly dominated by 
the faint-pulsar branch of $\mathcal{S}(L)\left(L_{\min }<L<L_{1}\right)$. The relevant analytic expressions are given for the variance:

$$
\begin{aligned}
\frac{\left[\left\langle N_{\mathrm{est}}^{2}\right\rangle-\left\langle N_{\mathrm{est}}\right\rangle^{2}\right]^{1 / 2}}{\left\langle N_{\mathrm{est}}\right\rangle}= & (p-1)^{1 / 2}\left(p+\frac{1}{2}\right)^{-1 / 2} L_{\min }^{(p-1) / 2} L_{2}^{1 / 2} \\
& \times\left[L_{\mathrm{min}}^{-(p+1 / 2)} L_{1}^{1 / 2}-L_{1}^{-p}\right]^{1 / 2} N_{G}^{-1 / 2}
\end{aligned}
$$

and the skewness:

$$
\begin{aligned}
\frac{\left\langle N_{\mathrm{est}}^{3}\right\rangle-3\left\langle N_{\mathrm{est}}\right\rangle\left\langle N_{\mathrm{est}}^{2}\right\rangle+2\left\langle N_{\mathrm{est}}\right\rangle^{3}}{\left[\int d L \phi(L) \mathcal{S}(L)\right]^{3 / 2}}= & (p-1)^{-1 / 2}(p+2)^{-1}\left(p+\frac{1}{2}\right)^{3 / 2} \\
& \times L_{\min }^{(1-p) / 2} L_{1} L_{2}^{1 / 2}\left[L_{\min }^{-(p+2)}-L_{1}^{-(p+2)}\right] \\
& \times\left[L_{\min }^{-(p+1 / 2)} L_{1}^{1 / 2}-L_{1}^{-p}\right]^{-3 / 2} N_{G}^{-1 / 2}
\end{aligned}
$$

where $N_{G}$ is given by equation (18) as a function of $p, L_{\min }, L_{1}, L_{2}$, and is proportional to $<N_{\text {obs }}>$.

For our standard case (model \# 1 in Table 1), we obtain from equations (A15), (A16), and (19):

$$
\frac{\left[\left\langle N_{\mathrm{est}}^{2}\right\rangle-\left\langle N_{\mathrm{est}}\right\rangle^{2}\right]^{1 / 2}}{\left\langle N_{\mathrm{est}}\right\rangle} \simeq 4\left\langle N_{\mathrm{obs}}\right\rangle^{-1 / 2}
$$

and

$$
\frac{\left\langle N_{\mathrm{est}}^{3}\right\rangle-3\left\langle N_{\mathrm{est}}\right\rangle\left\langle N_{\mathrm{est}}^{2}\right\rangle+2\left\langle N_{\mathrm{est}}\right\rangle^{3}}{\left[\int d L \phi(L) \mathcal{S}(L)\right]^{3 / 2}} \simeq 6\left\langle N_{\mathrm{obs}}\right\rangle^{-1 / 2} .
$$

Using our Monte Carlo simulations we were able to confirm our analytical results for both the variance and the skewness to an accuracy better than 1\%. A comparison of numerical and analytical results is shown in Figure 7, for our standard case. We note that the above analytic expressions can be used to update the results on the variance and the skewness of the $N_{\text {est }}$-distribution as our knowledge of the luminosity function improves.

\section{REFERENCES}

Arzoumanian, Z., et al. 1996, ApJ, 470, 1111

Arzoumanian, Z., Cordes, J. M., \& Wasserman, I. 1998, ApJ, 520, 696

Bailes, M. 1996, in Compact Stars in Binaries, IAU Symp. No. 165, eds. J. van Paradijs, E. P. J. van den Heuvel and E. Kuulkers (Kluwer Academic Publishers, Dordrecht), 213

Belczynski, K., Bulik, T., \& Rudak, B. 1999, to appear in the Proceedings of the 5th Huntsville Gamma Ray Burst Symposium (October 1999), ed. R.M. Kippen (AIP) 
Belczynski, K., \& Kalogera, V. 2001, ApJ, in press astro-ph/0012172

Belczynski, K., Kalogera, V., \& Bulik, T. 2000, ApJ, in preparation

Bethe, H., \& Brown, G. E. 1998, ApJ, 506, 780

Bhattacharya, D. \& van den Heuvel, E. P. J. 1991, Phys. Rep., 203, 1

Biggs, J.D. 1990, MNRAS, 245, 514

Blanton, M., et al. 2000, ApJ, to be submitted

Blitz, L. 1997, in "CO: Twenty-Five Years of Millimeter-Wave Spectroscopy", eds. W.B. Latter, et al. (Kluwer Academic Publishers), 11

Brandt, N. \& Podsiadlowski, P. 1995, MNRAS,

Bulik, T., Belczynski, K., \& Zbijewski, W. 1999, MNRAS, 309, 629

Cordes, J. M., Chernoff, D. F. 1997, ApJ, 482, 971

Curran, S. J., \& Lorimer, D. R. 1995, MNRAS, 276, 347

Dehnen, W., \& Binney, J.J. 1998, MNRAS, 298, 387

deJong, R.S., \& van der Kruit, P.C. 1994,A\&AS, 106, 451

Drimmel, R., Spergel, D.N. 2000, ApJ, to be submitted

Efstathiou, G., Ellis, R.S., \& Peterson, B.A. 1988, MNRAS, 232, 431

Eichler, D., et al. 1989, Nature, 340, 126

Evans, T. et al. 1999, to appear in the proceedings of the XXXIVth Rencontres de Moriond on "Gravitational Waves and Experimental Gravity", Les Arcs, France

Feast, M.W., \& Whitelock, P.A. 1997, MNRAS, 291, 683

Freudenreich, H.T. 1998, ApJ, 492, 495

Fryer, C. L. 1999, ApJ, 522, 413

Fryer, C. L., Benz, W., Herant, M. 1996, ApJ, 460, 801

Fryer, C. L., Burrows, A., Benz, W. 1998, ApJ, 496, 333

Fryer, C.L. \& Kalogera, V. 1997, ApJ, 489, 244

Fryer, C. L., Woosley, S. E., \& Hartmann, D. H. 1999, ApJ, 526, 152 
Grishchuk, L.P., et al. 2001, Physics-Uspekhi, 171, 3 [astro-ph/0008481

Gustafson, E., Shoemaker, D., Strain, K., and Weiss, R., LSC White Paper on Detector Research and Development (LIGO-Project document, September 11, 1999).

Hartman, J.W. 1997, A\&A, 322, 127

Hills, J. G. 1983, ApJ, 267, 322

Hulse, R. A., \& Taylor, J. H. 1975, ApJ, 201, L55

Johnston, S. 1994, MNRAS, 268, 595

Junker, W., \& Schaefer, G. 1992, MNRAS, 254, 146

Kalogera, V. 1996, ApJ, 471, 352

- 2000a, Proceedings of the Third Edoardo Amaldi Conference on Gravitational Waves, ed. S. Meshkov, p. 41

. 2000b, ApJ, 541, 319

Kalogera, V., \& Belczynski, K. 2000, to appear in the conference proceedings The influence of binaries on stellar population studies, eds. D. Vanbeveren \& W. van Rensbergen

Kalogera, V., \& Lorimer, D. R. 2000, ApJ, 530, 890

Kalogera, V., \& Webbink, R.F. 1998, ApJ, 493, 351

Karastergiou, A., et al. 2000, in "Pulsar Astronomy - 2000 and Beyond", IAU Colloq. 177, eds. M. Kramer, N. Wex, R. Wielebinski, p. 125

Kaspi, V. M., et al. 2000, ApJ, in press (astro-ph/0005214)

Kramer. M. 1998, ApJ, 509, 856

Kuijken, K., \& Gilmore, G. 1989, MNRAS, 239, 571

Lacey, C.G., \& Fall, S.M. 1985, ApJ, 290, 154

Lin, H., et al. 1996, ApJ, 464, 60

Lipunov, V. M., Postnov, K. A., \& Prokhorov, M. E. 1997, MNRAS, 288, 245

Lorimer, D.R., et al. 1993, MNRAS, 263, 403

Loveday, J., et al. 1992, ApJ, 390, 338

Lyne, A.G., \& Bailes, M. 1990, MNRAS, 246, L15 
Lyne, A. G., Manchester, R. N., \& Taylor, J. H. 1985, MNRAS, 213, 613

Lyne, A.G., \& Manchester, R.N. 1988, MNRAS, 234, 477

Lyne, A.G, \& Graham-Smith, F. 1998, in Pulsar Astronomy (Cambridge: Cambridge University Press), p. 156

Lyne, A. G. et al. 2000, MNRAS, 312, 698

Madau, P., Pozzetti, L., Dickinson, M. 1998, ApJ, 498, 106

Manchester, R.N., \& Taylor, J.H. 1977, in Pulsars (San Francisco: W.H. Freeman and Co.), Ch. 10

Narayan, R. 1987, ApJ, 319, 162

Narayan, R., Paczyński, B., \& Piran, T. 1992, ApJ, 395, L83

Narayan, R., Piran, T., \& Shemi, A. 1991, ApJ, 379, L17

Narayan, R., \& Vivekanand, M. 1983, A\&A, 122, 45

Nice, D.J., Sayer, R.W., \& Taylor, J.H. 1996, ApJ, 466, L87

Paczynski, B. 1986, ApJ, 308, L43

Paczynski, B. 1990, ApJ, 348, 485

Peters, P.C., \& Mathews, J. 1963, Phys. Rev. D, 131, 435

Phinney, E. S. 1991, ApJ, 380, L17

Portegies-Zwart, S. F., \& Yungel'son, L. R. 1998, A\&A, 332, 173

Prince, T.A., et al. 1991, ApJ, 374, L41

Radhakrishnan, V., \& Cooke, D.J. 1969, Astrophys. Lett., 3, 225

Rasio, F.A., \& Shapiro, S.L. 1999, Classical Quantum Gravity, 16, R1

Ritchings, R.T. 1976, MNRAS, 176, 249

Rosswog, S. et al. 1999, A\&A, 341, 499

Saunders, W., et al. 1990, MNRAS, 242, 318

Shapiro, S. L. \& Teukolsky, S. A. 1983, Black Holes, White Dwarfs, and Neutron Stars (John Wiley \& Sons), p. 477-478

Soifer, B.T., \& Neugebauer, G. 1991, AJ, 101, 354 
Spruit, H., \& Phinney, E.S. 1998, Nature, 393, 139

Stairs, I. H. et al. 1998, ApJ, 505, 352

Stairs, I., et al. 2000, in "Pulsar Astronomy - 2000 and Beyond", IAU Colloq. 177, eds. M. Kramer, N. Wex, R. Wielebinski, p. 121

Sullivan, M., et al. 2000, MNRAS, 312, 442

Taylor, J.H. 1999, Proc. XXXIVth Rencontres de Moriond "Gravitational Waves and Experimental Gravity", in press

Taylor, J.H. \& Cordes, J.M. 1993, ApJ, 411, 674

Taylor, J. H., \& Weisberg, J. M. 1982, ApJ, 253, 908

- 1989, ApJ, 345, 434

Thorsett. S. E., \& Chakrabarty, D. 1999, ApJ, 512, 288

van den Heuvel, E. P. J., \& Lorimer, D. R. 1996, MNRAS, 283, 37

van Kerkwijk, M.H., \& Kulkarni, S.R. 1999, ApJ, 516, L25

van der Kruit, P.C. 1987, in "The Galaxy", eds. G. Gilmore \& B. Carswell (Dordrecht: Reidel), p. 27

Weisberg, J.M., \& Taylor, J.H. 2000, in "Pulsar Astronomy - 2000 and Beyond", IAU Colloq. 177, eds. M. Kramer, N. Wex, R. Wielebinski, p. 127

Wex, N., Kalogera, V., \& Kramer, M. 2000, ApJ, 528, 401

Wolszczan, A. 1991, Nature, 350, 688

Yasuda, N., Fukugita, M., \& Okamura, S. 1997, ApJS, 108, 417 


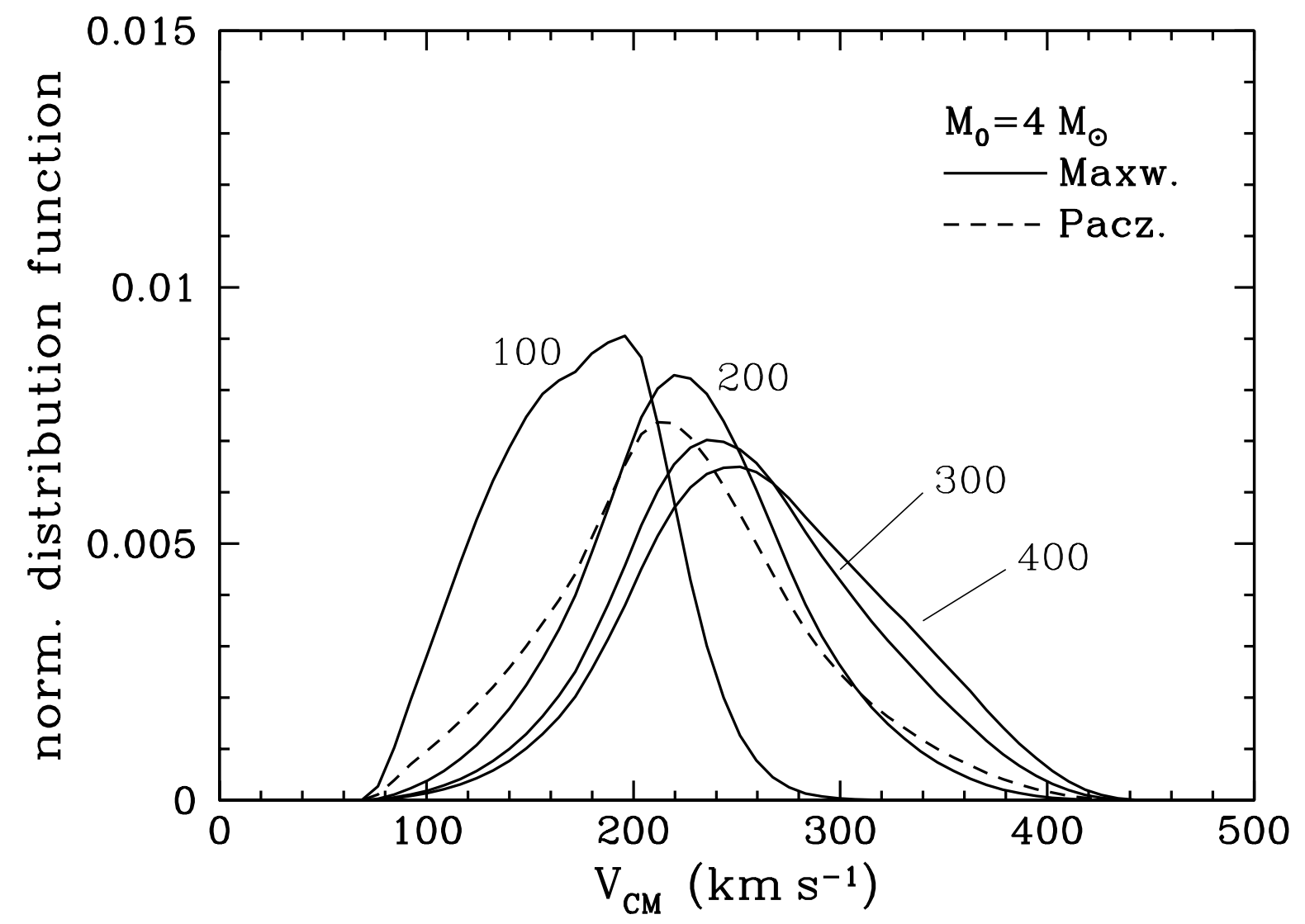

Fig. 1.- Probability distributions $f\left(V_{\mathrm{CM}}\right) d V_{\mathrm{CM}}$ of center-of-mass velocities $V_{\mathrm{CM}}$ of coalescing NS-NS binaries just after the second supernova explosion, shown for a specific helium-star mass $\left(M_{0}=4 \mathrm{M}_{\odot}\right)$. Solid lines correspond to models with a Maxwellian kick-magnitude distribution with $\sigma=100,200,300,400 \mathrm{~km} \mathrm{~s}^{-1}$, and the dashed line corresponds to a "Paczynski-like" kickmagnitude distribution (see text). NS kicks are assumed to be isotropic. 


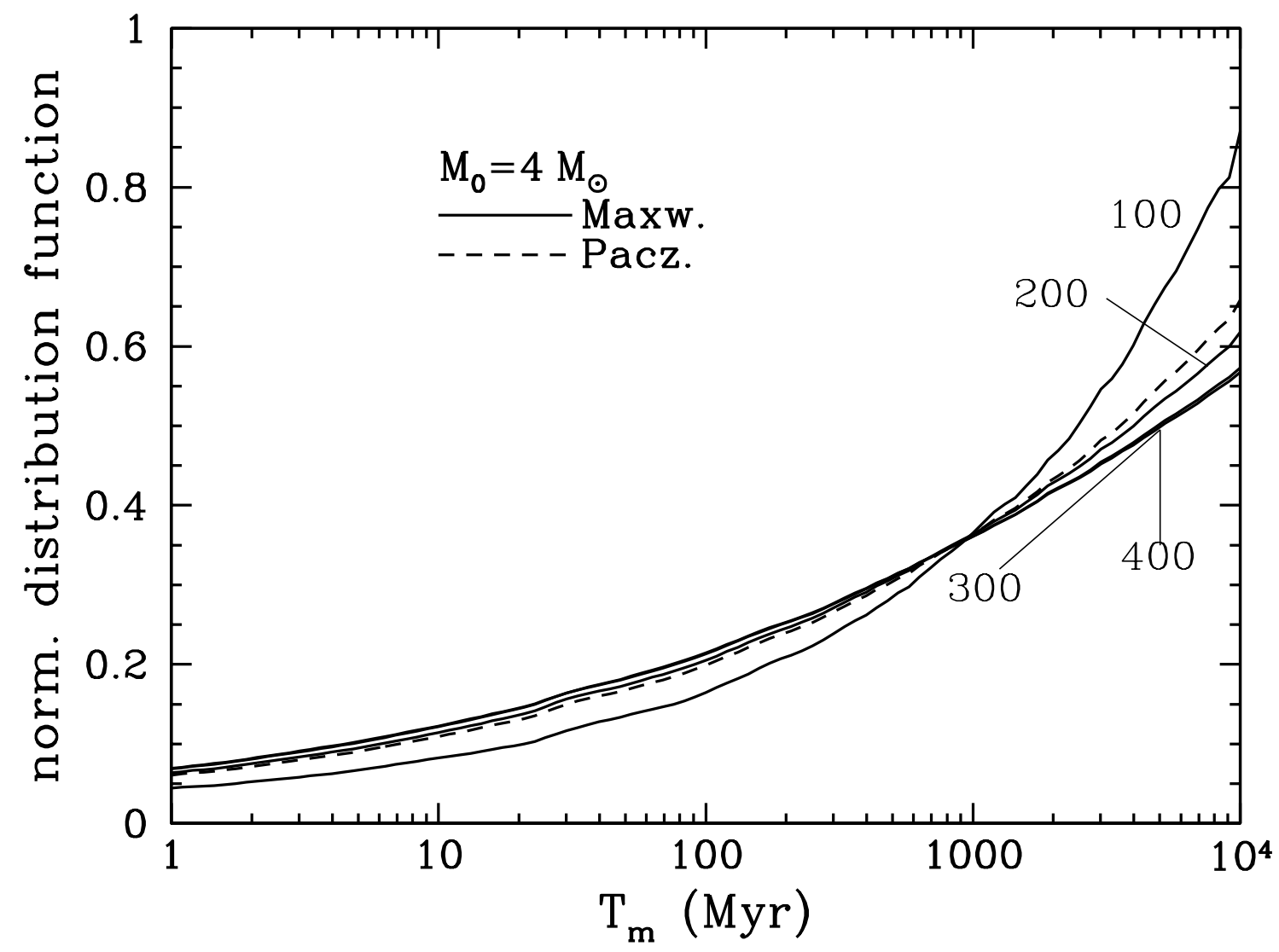

Fig. 2.- Probability distributions $\left.f\left(\log T_{m}\right) d \log T_{m}\right)$ of merger time scales $T_{m}$ of coalescing NS-NS binaries, for a specific helium-star mass $\left(M_{0}=4 \mathrm{M}_{\odot}\right)$. Line coding is as in Figure 1. 


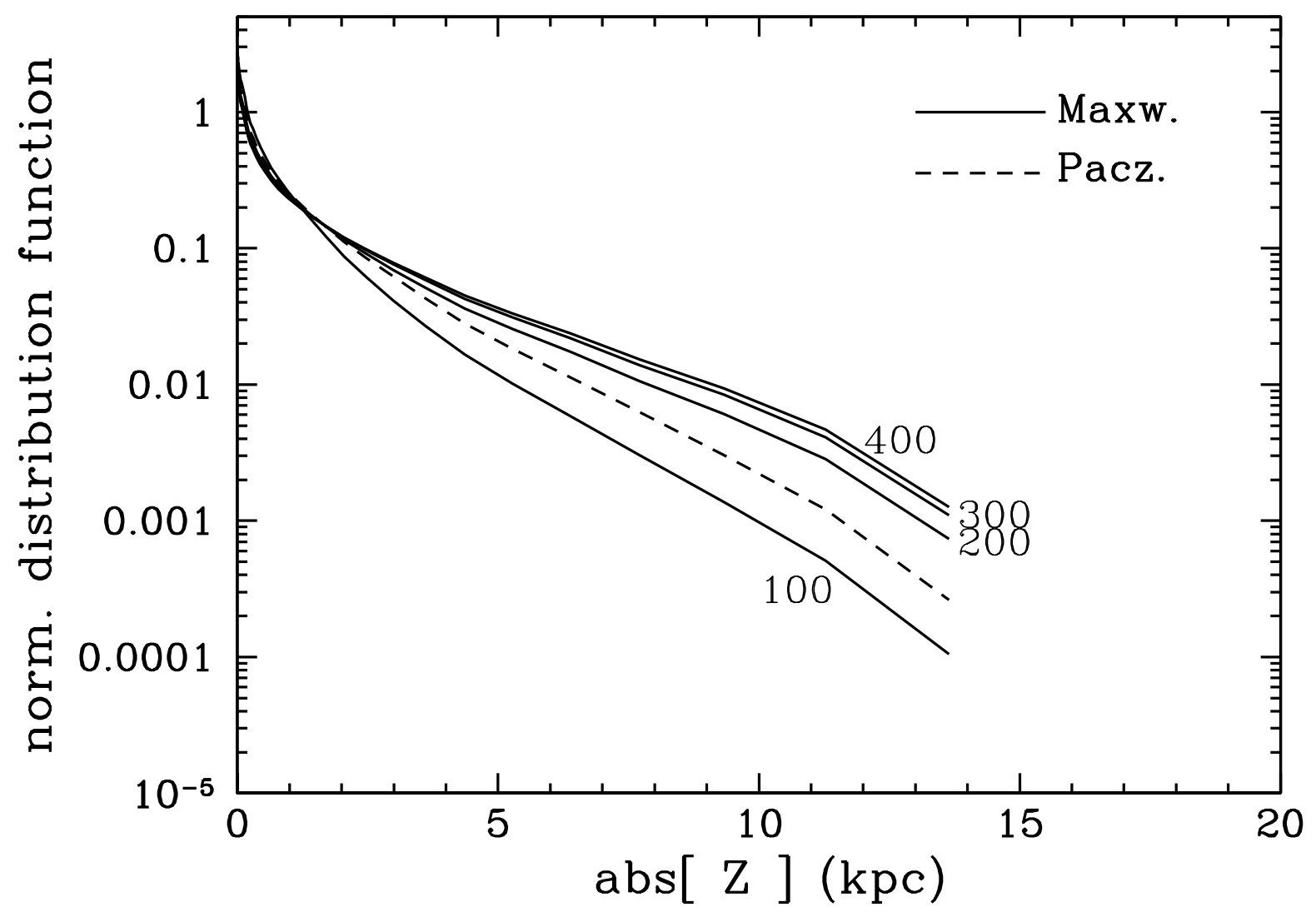

Fig. 3. - Probability distributions $f(|Z|) d Z$ of absolute vertical distance $Z$ from the Galactic plane of coalescing NS-NS binaries at present. A constant star-formation rate has been assumed for the Galaxy. Line coding is as in Figure 1. 


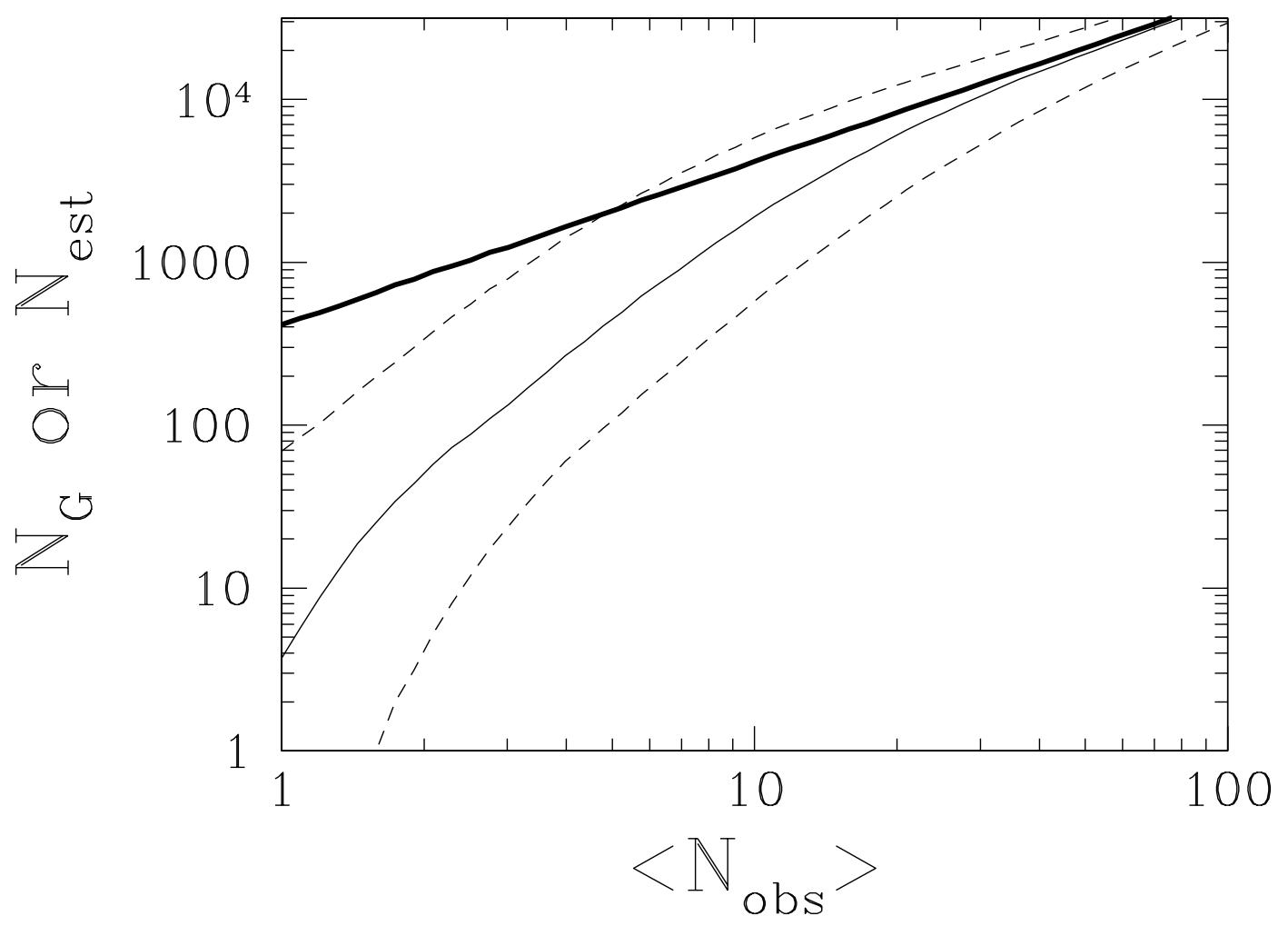

Fig. 4.- Total number $N_{G}$ of pulsars in the Galaxy (thick solid line) and statistical properties of the distribution of the estimated pulsar number $N_{\text {est }}$ (median: thin, solid line; first quartile: lower thin dashed line; third quartile: upper thin dashed line) as a function of the mean number of objects in the observed samples. Note that the mean estimated number $\left\langle N_{\text {est }}\right\rangle$ is equal to the total number $N_{G}$ in the model. Curves are shown for our standard case: $p=2, L_{\min }=1 \mathrm{mJy} \mathrm{kpc}^{2}$, $L_{1}=30 \mathrm{mJy} \mathrm{kpc}^{2}$, and $L_{2}=3000 \mathrm{mJy} \mathrm{kpc}^{2}$ (see text), which corresponds to Model \#1 in Table 1. 


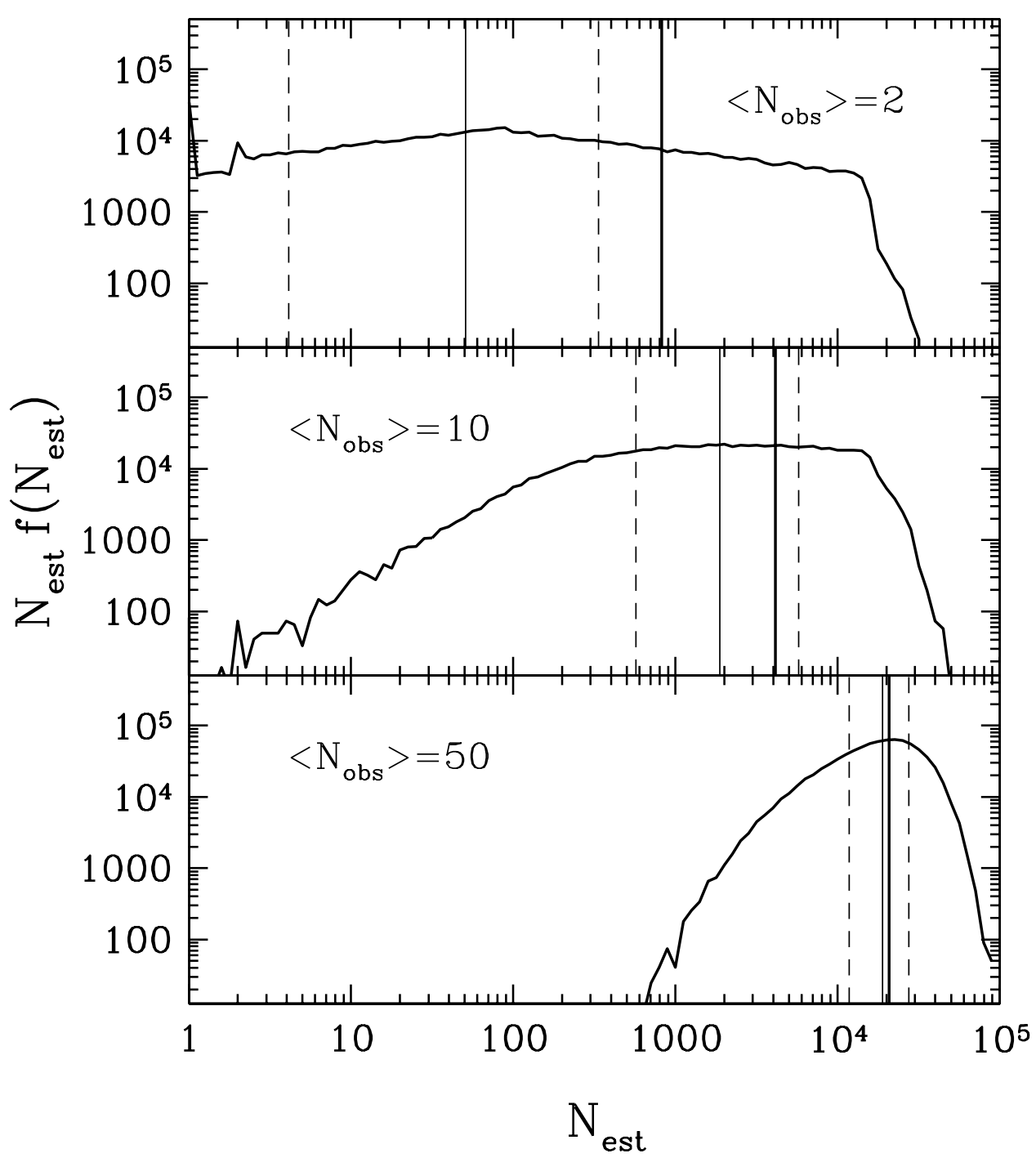

Fig. 5.- Distribution functions $f\left(N_{\text {est }}\right) d N_{\text {est }}$ of the estimated number $N_{\text {est }}$ of pulsars for $<N_{\text {obs }}>=2,10,50$ (top, middle, and bottom panels, respectively). Vertical lines mark the mean (thick solid line), median (thin solid line), and first and third quartiles (left and right dashed lines, respectively) values of the distributions. Results are shown for model number 1 (see Table 1). 


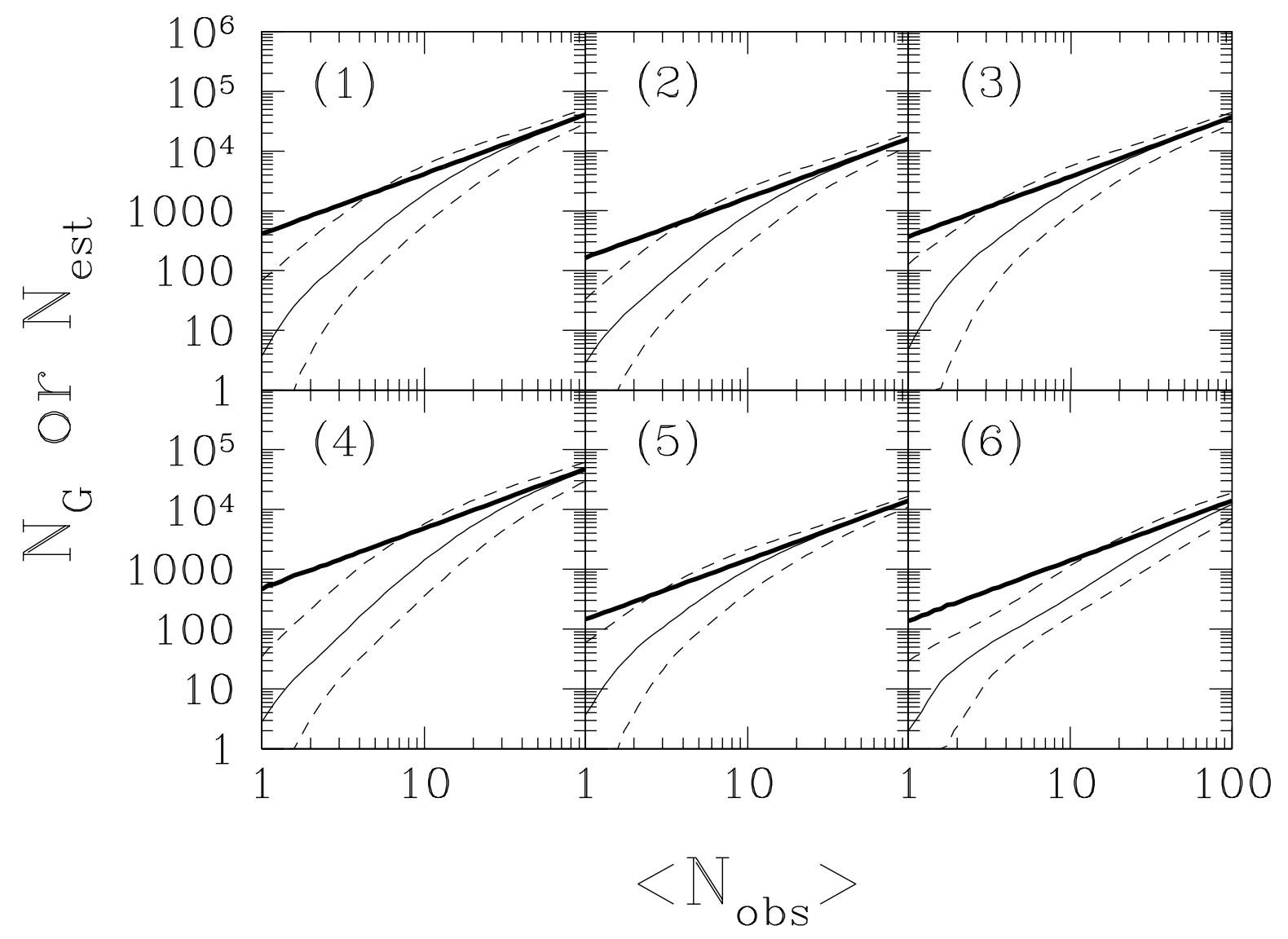

Fig. 6. - Same as in Figure 4. Each panel shows curves for one of the models listed in Table 1. Models are shown according to their model number (see Table 1) starting at the top left corner and ending at the bottom right corner. 


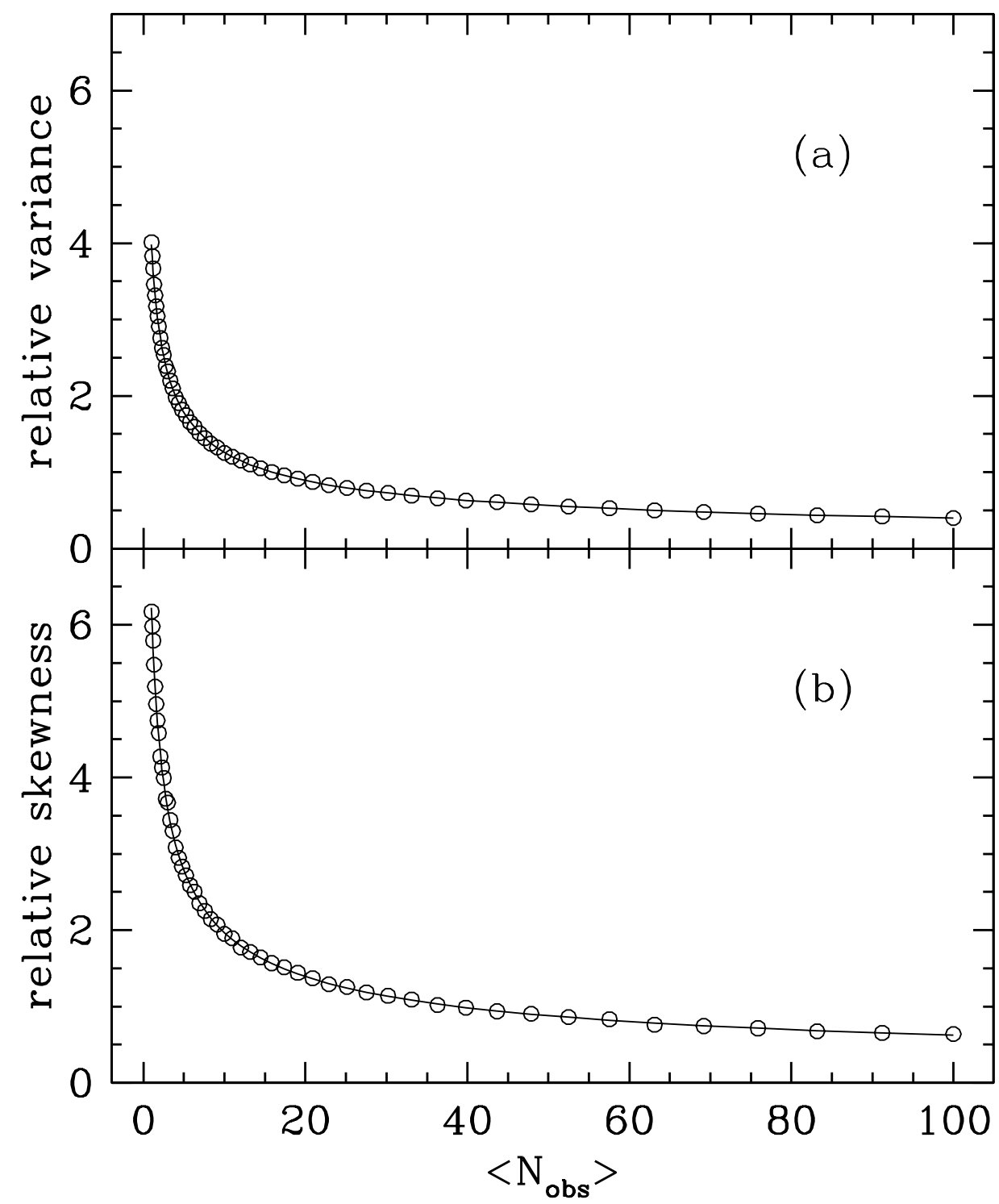

Fig. 7.- Moments of the $N_{\text {est }}$-distribution: (a) the variance relative to the mean and (b) the skewness relative to the variance as a function of the mean number of observed objects, for our standard case (model \# 1 in Table 1). The points are the results of our Monte Carlo simulations and the solid curve is the analytical results (equations [A16] and [A17]). The agreement is better than $1 \%$. 
Table 1. Correction Factors based on Distribution Properties of the Estimated Total Number of Pulsars $N_{\text {est }}$ based on Model Observed Samples

\begin{tabular}{ccccccccccccc}
\hline \hline \multicolumn{1}{c}{ Model Parameters } & \multicolumn{1}{c}{$<N_{\text {obs }}>=2^{\mathrm{a}}$} & \multicolumn{3}{c}{$<N_{\text {obs }}>=3^{\mathrm{a}}$} \\
Number & $\mathrm{p}^{\mathrm{b}}$ & $L_{\min }{ }^{\mathrm{c}}$ & $L_{1}{ }^{\mathrm{c}}$ & $L_{2}{ }^{\mathrm{c}}$ & $N_{G} \mathrm{~d}$ & $\frac{N_{G}}{\operatorname{Median}} \mathrm{e}$ & $\frac{N_{G}}{25 \%} \mathrm{f}$ & $\frac{N_{G}}{75 \%} \mathrm{~g}$ & $N_{G} \mathrm{~d}$ & $\frac{N_{G}}{\operatorname{Median}} \mathrm{e}$ & $\frac{N_{G}}{25 \%}{ }^{\mathrm{f}}$ & $\frac{N_{G}}{75 \%} \mathrm{~g}$ \\
\hline 1 & 2 & 1 & 30 & 3000 & 830 & 16 & 200 & 2.5 & 1245 & 9 & 52 & 1.5 \\
2 & 2 & 1 & 30 & 1000 & 325 & 13 & 107 & 2 & 490 & 7.5 & 35 & 1.3 \\
3 & 2 & 1 & 10 & 3000 & 745 & 8.5 & 143 & 1.5 & 1115 & 5 & 31 & 1 \\
4 & 2 & 1 & 100 & 3000 & 970 & 36 & 305 & 4.5 & 1450 & 20 & 102 & 2.5 \\
5 & 2 & 3 & 30 & 3000 & 290 & 7 & 75 & 1.3 & 430 & 4 & 21 & 0.95 \\
6 & 1.8 & 1 & 30 & 3000 & 270 & 12 & 120 & 3 & 400 & 8 & 32 & 2.8 \\
\hline
\end{tabular}

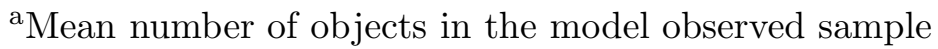

${ }^{\mathrm{b}}$ Power-law index of the pulsar luminosity function $\left(\propto L^{-p}\right)$

${ }^{\mathrm{c}}$ Break points in scale factor dependence on luminosity in units of mJy kpc ${ }^{2}$ (see eq. [16])

dModel total number of pulsars in the Galaxy

${ }^{\text {e}}$ Median of the $N_{\text {est }}$ distribution

${ }^{\mathrm{f}}$ First quartile of the $N_{\text {est }}$ distribution

${ }^{\text {g }}$ Third quartile of the $N_{\text {est }}$ distribution 